TRANSACTIONS OF THE

AMERICAN MATHEMATICAL SOCIETY

Volume 187, Issue 1, 1974

\title{
THE THEORY OF $Q$-RINGS
}

BY

\section{EBEN MATLIS}

ABSTRACT. An integral domain $R$ with quotient field $Q$ is defined to be a $Q$-ring if $\operatorname{Ext}_{R}^{1}(Q, R) \simeq Q$. It is shown that $R$ is a $Q$-ring if and only if there exists an $R$-module $A$ such that $\operatorname{Hom}_{R}(A, R)=0$ and $\operatorname{Ext}_{R}^{1}(A, R) \simeq Q$. If $A$ is such an $R$-module and $t(A)$ is its torsion submodule, then it is proved that $A / t(A)$ necessarily has rank one. There are only three kinds of $Q$-rings, namely, $Q_{0^{-}}, Q_{1}$, or $Q_{2}$-rings. These are described by the fact that if $R$ is a $Q$-ring, then $K=Q / R$ can only have 0,1 , or 2 proper $h$-divisible submodules. If $H$ is the completion of $R$ in the $R$-topology, then $R$ is one of the three kinds of $Q$ rings if and only if $H \otimes_{R} Q$ is one of the three possible kinds of 2-dimensional commutative $Q$-algebras. Examples of all three kinds of $Q$-rings are produced, and the behavior of $Q$-rings under ring extensions is examined. General conditions are given for a ring not to be a $Q$-ring. As an application of the theory, necessary and sufficient conditions are found for the integral closure of a noncomplete Noetherian domain to be a complete discrete valuation ring.

0 . Introduction. Throughout this paper $R$ will be an integral domain with quotient field $Q(Q \neq R)$, and $K$ will be the $R$-module $Q / R$. We shall say that $R$ is a $Q$-ring if $\operatorname{Ext}_{R}^{1}(Q, R) \cong Q$. The motivation for studying $Q$-rings arises out of a problem in singular cohomology theory posed originally by D. M. Kan and G. W. Whitehead [4]. They asked if there could exist an Abelian group $A$ such that $\operatorname{Hom}_{Z}(A, Z)=0$ and $\operatorname{Ext}_{Z}^{1}(A, Z) \cong Q$ (where $Z$ is the ring of integers and $Q$ is the field of rational numbers). They answered this question in the negative by an argument involving the lack of countability of the Abelian group $\operatorname{Ext}_{Z}^{1}(Q, Z)$. Further results along this line were proved by R. J. Nunke and J. J. Rotman [12], who analyzed the structure of singular cohomology groups. More recently $\mathrm{C}$. U. Jensen has studied the structure of $\operatorname{Ext}_{R}^{1}(A, R)$ in [2]. In [3] he examined the dimensionality of $\operatorname{Ext}_{R}^{1}(Q, R)$ as a vector space over $Q$, obtaining conditions for this dimension to be finite.

Our approach will be to study the class of integral domains $R$ that have an $R$-module $A$ such that $\operatorname{Hom}_{R}(A, R)=0$ and $\operatorname{Ext}_{R}^{1}(A, R) \cong Q$. Of course a $Q$-ring

Received by the editors August 21, 1972.

AMS (MOS) subject classifications (1970). Primary 13G05, 18G15; Secondary 13C05, $13 \mathrm{C10}$.

Key words and phrases. $\operatorname{Ext}_{R}^{1}(Q, R) \simeq Q, R$-topology, completion, cotorsion, $h$-divisible, torsion, full ring of quotients, strongly compatible ring extensions, integral extensions, minimal prime ideals, Noetherian domains of Krull dimension 1. 
is such an integral domain. We shall prove that conversely, if an integral domain has such a module then it is a $Q$-ring. We shall give examples to show that $Q$-rings exist-there are even discrete, rank one valuation rings (of characteristic 2) and nondiscrete rank one valuation rings (of characteristic 0 ) that are $Q$-rings (a fact already noted by Jensen). On the other hand we shall show that there are many kinds of integral domains that cannot be $Q$-rings, thus generalizing the results of Kan and Whitehead. Our approach, however, will be wholly homological in nature, and will not depend in any way upon countability arguments.

The property of being a $Q$-ring is essentially a property determined by $H$, the completion of $R$ in the $R$-topology. It is somewhat surprising, but there is a good deal of structure involved in the theory of $Q$-rings-particularly in the structure of $H$ and of the module $K$. We shall discover that there are three kinds of $Q$-rings, which we have called $Q_{0^{-}}, Q_{1}^{-}$, and $Q_{2}$-rings, depending upon whether the number of proper, nonzero, $b$-divisible submodules of $K$ is 0,1 , or 2 . At the end this paper we shall give examples of each of these three kinds of $Q$-rings. It is well known that there are only three possible kinds of two-dimensional commutative $Q$-algebras, and we shall show that the full ring of quotients of $H$ can be any one of these three, depending upon which type of $Q$-ring we are dealing with. It is from a detailed study of these three kinds of $Q$-rings that we can determine that certain kinds of integral domains cannot be $Q$-rings.

We shall examine the problem of making new $Q$-rings from old ones, and we shall show that certain kinds of integral extensions of a $Q$-ring are again $Q$-rings. The theory makes contact with both the theory of 1-dimensional Noetherian domains, and the theory of rings of type I (the rings which play such an important part in the theory of $D$-rings [9]).

For the convenience of the reader we shall present at this point a list of most of the definitions used in this paper. We shall assume that the reader is familiar with the elementary concepts of homological algebra, and we shall not define such terms as exact sequence, injective, Ext, homological dimensions, etc.

Definitions. (1) An $R$-module is said to be torsion-free if all of the multiplications on the module by the nonzero elements of $R$ are monomorphisms.

(2) An $R$-module is said to be a torsion module if it has no nonzero torsionfree submodules.

(3) An $R$-module $A$ has a unique largest torsion submodule $t(A)$ that contains every other torsion submodule of $A . A / t(A)$ is torsion-free.

(4) An $R$-module $T$ is said to be torsion of bounded order if it is not a faithful $R$-module; that is, if there exists a nonzero element $r \in R$ such that $r T=0$.

(5) An $R$-module is said to be divisible if all of the multiplications on the module by the nonzero elements of $R$ are epimorphisms.

(6) An $R$-module is said to be a reduced module if it has no nonzero divisible submodules. 
(7) An $R$-module $A$ has a unique largest divisible submodule $d(A)$ that contains every other divisible submodule of $A . A / d(A)$ is reduced.

We note that if $A$ is any $R$-module, then $\operatorname{Ext}_{R}^{n}(Q, A)$ is torsion-free and divisible for all $n \geq 0$; and $\operatorname{Hom}_{R}(K, A)$ is torsion-free and cotorsion (see [7]).

(8) For the purposes of homological algebra a more useful concept than divisible is that of $b$-divisible. An $R$-module is said to be $b$-divisible if it is a homomorphic image of an injective $R$-module. It is said to be $b$-reduced if it has no nonzero $b$-divisible submodules. An $R$-module $A$ has a unique largest $b$-divisible submodule $b(A)$ that contains every other $b$-divisible submodule of $A$. Unfortunately, however, $A / b(A)$ is not necessarily $b$-reduced. $(A / b(A)$ is always $b$-reduced if and only if the homological dimension of $Q$ is 1.$)$

(9) An $R$-module $A$ is said to be a cotorsion $R$-module if $\operatorname{Hom}_{R}(Q, A)=0$ and $\operatorname{Ext}_{R}^{1}(Q, A)=0$.

(10) The $R$-topology on an $R$-module $A$ is defined by taking the submodules of the form $I A$, where $I$ is a nonzero ideal of $R$, as a basis of neighborhoods of 0 in $A$. This is a uniform topology, and thus every $R$-module has a completion in this topology. An $R$-module is said to be complete in the R-topology if it is isomorphic to its completion. A complete $R$-module is a cotorsion $R$-module; and a torsionfree $R$-module is complete if and only if it is a cotorsion $R$-module. In particular, $R$ is complete if and only if $\operatorname{Ext}_{R}^{1}(Q, R)=0$.

(11) The completion of $R$ in the $R$-topology is denoted by $H . H$ is a commutative ring and is naturally isomorphic to $\operatorname{Hom}_{R}(K, K)$. We have a natural imbedding of $R$ into $H$, and $H / R$ is isomorphic to $\operatorname{Ext}_{R}^{1}(Q, R)$ and hence is torsionfree and divisible.

(12) $R$ is said to be an b-local ring if each nonzero ideal of $R$ is contained in only a finite number of maximal ideals of $R$, and each nonzero prime ideal of $R$ is contained in only one maximal ideal of $R$.

(13) A quasi-local ring is a ring with only one maximal ideal.

(14) If $A$ is a torsion-free $R$-module, we shall define the $Q$-rank of $A$ to be the dimension of $Q \otimes_{R} A$ as a vector space over $Q$. This is commonly referred to merely as the rank of $A$, but we wish to avoid any confusion with the notion of the rank of an ideal.

1. Preliminary results. In this section we shall prove a number of unrelated results that we shall need for the development of the theory of $Q$-rings, but whose applicability is far wider and more general than this theory.

Theorem 1.1. If $T$ is a torsion $R$-module, then there is a unique $H$-module structure on $T$ extending that of $R$. Every $R$-submodule of $T$ is also an $H$-submodule of $T$. If $B$ is any $H$-module, then $\operatorname{Hom}_{R}(T, B)=\operatorname{Hom}_{H}(T, B)$. 
Proof. Since $H / R$ is torsion-free and divisible by [7, Proposition 5.2], we have an isomorphism $\eta: T \rightarrow H \otimes_{R} T$, and thus $T$ has an $H$-module structure extending that of $R$ defined by $b x=\eta^{-1}(b \otimes x)$ for $b \in H$ and $x \in T$. Suppose that we have another $H$-module structure on $T$ extending that of $R$ and denoted by $b \cdot x$. There is a nonzero element $r \in R$ such that $r x=0$. Because $H / R$ is divisible there are elements $g \in H$ and $s \in R$ such that $b=r g+s$. Thus $b x=$ $s x=b \cdot x$, and the two $H$-structures are identical. It is also clear that every $R$ submodule of $T$ is also an $H$-submodule of $T$.

Let $f$ be an $R$-homomorphism from $T$ to $B$. With the notation of the preceding paragraph we have $f(b x)=f(s x)=s f(x)=b f(x)-g f(r x)=b f(x)$, and thus $f$ is an H-homomorphism.

Theorem 1.2. Let $T$ be an R-module. Then $T$ is torsion and b-divisible if and only if for every $x \in T$ there is an R-homomorphism $f: K \rightarrow T$ such that $x \in f(K)$.

Proof. Suppose that $T$ is a torsion, $b$-divisible $R$-module. Then there is an injective $R$-module $C$ and an $R$-homomorphism of $C$ onto $T$. Thus there is an $R$-homomorphism $\nu$ from $Q$ into $T$ such that $\nu(1)=x$. If $S=\operatorname{Ker} \nu$, then $S \neq 0$ because $T$ is a torsion $R$-module. Choose a nonzero element $q \in S$ and define $\eta: Q \rightarrow T$ by $\eta(y)=\nu(q y)$ for all $y \in Q$. Then $\eta\left(q^{-1}\right)=x$ and $\eta(1)=0$. Hence we have an induced $R$-homomorphism $f: K \rightarrow T$ such that $x \in f(K)$.

Conversely, suppose that for each $x \in T$ there is an $R$-homomorphism $f: K \rightarrow T$ such that $x \in f(K)$. Then $T$ is certainly a torsion $R$-module, and there is an $R$ homomorphism from a direct sum of copies of $Q$ onto $T$. Since a direct sum of copies of $Q$ is injective, $T$ is an $b$-divisible $R$-module.

Corollary 1.3. If $B$ and $C$ are b-divisible submodules of a torsion $R$-module, then $\operatorname{Hom}_{R}(K, B)=\operatorname{Hom}_{R}(K, C)$ if and only if $B=C$.

Proof. This is an immediate consequence of Theorem 1.2.

Theorem 1.4. Let $A$ be a commutative ring extension of $R$. Then the following statements are equivalent:

(1) $J \cap R \neq 0$ for every nonzero ideal $J$ of $A$.

(2) $A$ is an integral domain and the quotient field of $A$ is isomorpbic to $A \otimes_{R} Q$.

Proof. We let $R-\{0\}=S$, and identify $A \otimes_{R} Q$ with the ring of quotients $A_{S}$. Both (1) and (2) are then seen to be equivalent to the assertion that if $a$ is a nonzero element of $A$, then there exist nonzero elements $b \in A$ and $r \in R$ such that $b a=r$. 
Theorem 1.5. Let $A$ be a commutative ring extension of $R$ such that $J \cap R \neq 0$ for every nonzero ideal $J$ of $A$ and let $B$ be an $A$-module. Then $A$ is an integral domain, and the R-topology and the A-topology on B coincide. Furthermore, $B$ bas one of the following properties as an R-module if and only if it has the same property as an A-module: (1) torsion, (2) b-reduced, (3) cotorsion, (4) complete, (5) divisible, (6) b-divisible.

Proof. $A$ is an integral domain by Theorem 1.4. It is obvious that the $R$-topology and the $A$-topology on $B$ coincide. Thus (4) is true. (1) and (5) follow trivially from the proof of Theorem 1.4. By [1, Chapter VI, Proposition 4.1.3], we have $\operatorname{Ext}_{R}^{n}(Q, B) \cong \operatorname{Ext}_{A}^{n}\left(A \otimes_{R} Q, B\right)$ for all $n \geq 0$. Since $A \otimes_{R} Q$ is the quotient field of $A$ by Theorem 1.4, (2) and (3) are seen to be true. We have a commutative diagram:

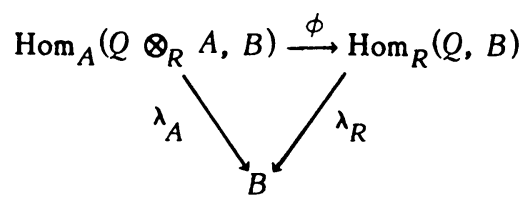

where $\phi$ is the canonical isomorphism: $\lambda_{R}$ is defined by $\lambda_{R}(f)=f(1)$ for $f \epsilon$ $\operatorname{Hom}_{R}(Q, B)$; and $\lambda_{A}$ is defined similarly. According to [7, Chapter II, p. 3], $B$ is $b$-divisible over $R$ (or over $A$ ) if and only if $\lambda_{R}\left(\right.$ or $\lambda_{A}$ ) is an epimorphism. Thus we see that (6) is true.

Corollary 1.6. Suppose that $A$ is an integral domain containing $R$ and that the quotient field of $A$ is algebraic over $Q$. Then the quotient field of $A$ is isomorphic to $A \otimes_{R} Q$, and thus all the statements of Theorem 1.5 are true for $R$ and $A$.

Proof. If $x$ is a nonzero element of $A$, then the minimum polynomial for $x$ over $Q$ has a nonzero constant term. From this it follows readily that if $J$ is a nonzero ideal of $A$, then $J \cap R \neq 0$. The corollary is now a consequence of Theorems 1.4 and 1.5 .

Theorem 1.7. If $A$ is a commutative ring extension of $R$, then the following statements are equivalent:

(1) $A=J+R$ and $J \cap R \neq 0$ for every nonzero ideal $J$ of $A$.

(2) $A / R$ is a divisible $R$-module, and $J \cap R \neq 0$ for every nonzero ideal $J$ of $A$.

(3) $A / J \cong R /(J \cap R)$ and $J=A(J \cap R)$ for every nonzero ideal $J$ of $A$.

Proof. (1) $\Rightarrow(2)$. If $a$ is a nonzero element of $R$, then $A=A a+R$, and hence $a(A / R)=A / R$. Thus $A / R$ is a divisible $R$-module. 
(2) $\Rightarrow(1)$. If $J$ is a nonzero ideal of $A$, choose a nonzero element $a \in J \cap R$. Then $a(A / R)=A / R$, and hence $A=A a+R \subset J+R \subset A$.

$(1) \Rightarrow(3)$. Let $J$ be a nonzero ideal of $A$; then $A / J=(J+R) / J \cong R /(J \cap R)$. Let $I=A(J \cap R)$; then $I$ is a nonzero ideal of $A$ and $I \subset J$. Thus $A=I+R$; and hence if $x \in J$, then $x=y+r$, where $y \in I$ and $r \in R$. Therefore, $r=x-y \in J \cap R \subset I$; and consequently $x \in I$. Thus we see that $J=I$.

$(3) \Rightarrow(1)$. Let $J$ be a nonzero ideal of $A$. Since $J=A(J \cap R)$, it follows that $J \cap R \neq 0$. Now $A / J$ is a cyclic $R$-module by assumption, and hence there is an element $a \in A$ such that $A=R a+J$. Thus $1=r a+j$, where $r \in R$ and $j \in J$. Therefore, we have $A=A r+J$. We also have $A r=R a r+J r$, and hence $A=R a r+J$. But $R r a+J=R+J$, and thus $A=R+J$.

Definition. If an integral domain extension of $R$ satisfies any one of the three equivalent conditions of Theorem 1.7 and is not a field, we shall say that it is a compatible ring extension of $R$.

Corollary 1.8. Let $A$ be a proper $R$-submodule of $Q$ containing $R$ ( $A$ not necessarily a ring). Then $A$ is a compatible ring extension of $R$ if and only if $A / R$ is a divisible $R$-module.

Proof. Suppose that $A / R$ is a divisible $R$-module. Let $q$ be a nonzero element of $A$; then $q=a / b$, where $a, b \in R$ and $b \neq 0$. Then $A=A b+R$, and hence $A q=A a+R q$. Since $A a$ and $R q$ are contained in $A$, we have $A q \subset A$. Thus $A$ is a ring. Clearly every nonzero ideal of $A$ has a nonzero intersection with $R$. Therefore, $A$ is a compatible ring extension of $R$ by Theorem 1.7. The converse statement also follows from Theorem 1.7.

Theorem 1.9. Let $A$ be a compatible ring extension of $R$. Then

(1) $A$ is an integral domain with quotient field $A \otimes_{R} Q$, and all of the statements of Theorem 1.5 are true for $R$ and $A$.

(2) A finitely generated torsion A-module is also a finitely generated torsion $R-$ module.

(3) If $R$ is a Noetherian ring, then $A$ is also a Noetberian ring.

(4) $N$ is a maximal (resp. prime) ideal of $A$ if and only if $P=N \cap R$ is a maximal (resp. prime) ideal of $R$. In this case $A_{P}=A_{N}$, and $A_{P}$ is a compatible ring extension of $R_{P}$. Thus if $R$ is a quasi-local ring, then $A$ is also quasi-local.

(5) Let $S$ be an $R$-submodule of $Q$ containing $R$. Then $A S=A+S$ and $A S / S$ is a divisible $R$-module. If $A \subset S$, then $S$ is an $A$-module. If $S$ is a proper subring of $Q$ containing $R$, then $A S / S$ is a divisible $S$-module, and if $A S$ is not a field, then $A S$ is a compatible ring extension of $S$.

(6) If $V$ is a valuation subring of $Q$ containing $R$ and $A \subset Q$, then either $A+V=Q$ or $A \subset V$. 
Proof. (1) This is a direct consequence of Theorem 1.4.

(2) A cyclic torsion $A$-module is a cyclic torsion $R$-module by Theorem 1.7. The general statement follows easily by induction on the number of generators.

(3) Theorem 1.7 gives an immediate proof of (3).

(4) Let $N$ be a nonzero ideal of $A$; then $A=R+N$ by Theorem 1.7. Hence we have a ring homomorphism of $R$ onto $A / N$ with kernel $N \cap R$; that is, $A / N$ and $R /(N \cap R)$ are isomorphic as rings. Hence $N$ is a maximal (resp. prime) ideal of $A$ if and only if $N \cap R$ is a maximal (resp. prime) ideal of $R$. If $M$ is the only maximal ideal of $R$, and $N$ is any maximal ideal of $A$, then we see that $M=N \cap R$, and hence $N=A(N \cap R)=A M$. Therefore, $N$ is the only maximal ideal of $A$. Hence if $R$ is quasi-local, $A$ is also quasi-local.

Let $N$ be a nonzero prime ideal of $A$, and let $P=N \cap R$. Then $A_{P} / R_{P}$ is a divisible $R_{P}$-module; and $A_{P}$ is not a field, since $A_{P} \subset A_{N}$. It is clear that every nonzero ideal of $A_{P}$ has a nonzero intersection with $R_{P}$. Thus $A_{P}$ is a compatible ring extension of $R_{P}$ by Theorem 1.7. Because $R_{P}$ is a quasi-local ring with maximal ideal $P R_{P}, A_{P}$ is a quasi-local ring with maximal ideal $P A_{P}$ by what has already been proved. Thus we have $N A_{P}=P A_{P}$, from which it follows immediately that $A_{N}=A_{P}$.

(5) If $q \in Q, q=a / b$ where $a, b \in R$ and $b \neq 0$, then because $A / R$ is a divisible $R$-module we have $A q=A a+R q$. Let $S$ be an $R$-submodule of $Q$ containing $R$; then if $q \in S$, we see that $A q \subset A+S$, and hence $A S \subset A+S$. Since $1 \in A \cap S$, we have the reverse inequality. Thus $A S / S \cong A /(A \cap S)$, and hence $A S / S$ is a homomorphic image of $A / R$. Therefore, $A S / S$ is a divisible $R$-module. If $A \subset S$, then $A S=A+S=S$, and hence $S$ is an $A$-module.

Suppose that $S$ is a proper subring of $Q$ containing $R$. It is obvious that every nonzero ideal of $A S$ has a nonzero intersection with $S$. Because $A S / S$ is a divisible $R$-module, it is also a divisible $S$-module by Theorem 1.5. Therefore, if $A S$ is not a field, then $A S$ is a compatible ring extension of $S$ by Theorem 1.7.

(6) Let $V$ be a valuation subring of $Q$ containing $R$, and suppose that $A \subset Q$. If $A+V \neq Q$, then $A V=A+V$ is a proper $V$-submodule of $Q$. Hence $(A V) / V$ is a torsion $V$-module of bounded order. By (5) $(A V) / V$ is a divisible $V$-module, and thus $A V=V$. Hence we have $A \subset V$.

Theorem 1.10. Let $S$ be a proper ring extension of $R$ in $Q$, and let $H(S)$ denote the completion of $S$ in the $S$-topology. Then we bave an exact sequence

$$
0 \rightarrow \operatorname{Hom}_{R}(K, S / R) \rightarrow H \stackrel{\phi}{\rightarrow} H(S) \rightarrow \operatorname{Ext}_{R}^{1}(K, S / R)
$$

where $\phi$ is a ring homomorphism.

Proof. As we have seen in the Introduction, the completion of $S$ in the $S$ topology is given by $H(S)=\operatorname{Hom}_{S}(Q / S, Q / S)$; and the completion of $R$ in the 
$R$-topology is given by $H=\operatorname{Hom}_{R}(K, K)$. Now $\operatorname{Hom}_{S}(Q / S, Q / S)=\operatorname{Hom}_{R}(Q / S, Q / S)$. For let $g \in \operatorname{Hom}_{R}(Q / S, Q / S)$ and $s \in S$. Define an $R$-homomorphism $\xi: Q / S \rightarrow Q / S$ by $\xi(x)=s g(x)-g(s x)$ for all $x \in Q / S$. Since $s=a / b$ where $a, b \in R$ and $b \neq 0$, we have $b \xi=0$. Since $Q / S$ is a divisible $R$-module, this implies that $\xi=0$, and thus $g$ is an $S$-homomorphism.

By Theorem 1.1, $Q / S$ is an $H$-module and $\operatorname{Hom}_{R}(Q / S, Q / S)=\operatorname{Hom}_{H}(Q / S, Q / S)$. Thus we have a ring homomorphism $\phi: H \rightarrow H(S)$ defined by $\phi(b)(x)=b x$ for all $b \in H$ and $x \in Q / S$. Let $\Pi$ be the canonical $R$-homomorphism of $K$ onto $Q / S$ with $\operatorname{Ker} \Pi=S / R$. Then we have a diagram:

$$
0 \rightarrow \operatorname{Hom}_{R}(K, S / R) \rightarrow \operatorname{Hom}_{R}(K, K) \stackrel{\eta}{\rightarrow} \operatorname{Hom}_{R}(K, Q / S) \stackrel{\delta}{\rightarrow} \operatorname{Ext}_{R}^{1}(K, S / R)
$$

where $\eta$ and $\lambda$ are induced by $\Pi$ and the top row is exact. Since $\Pi$ is an $H$-homomorphism by Theorem 1.1 , it is easily verified that the diagram commutes. Let $\beta=\delta \lambda$; because $\lambda$ is a monomorphism, it is easily checked that $\operatorname{Im} \phi=\operatorname{Ker} \beta$ and that $\operatorname{Ker} \phi=\operatorname{Hom}_{R}(K, S / R)$. This establishes the theorem.

Theorem 1.11. Let $D$ be a nonzero, torsion, $b$-divisible $R$-module such that $\operatorname{Hom}_{R}(K, D)$ bas finite $Q$-rank, and let $B$ be a proper $R$-submodule of $D$. Then

$$
Q \text {-rank }\left(\operatorname{Hom}_{R}(K, B)\right)<Q \text {-rank }\left(\operatorname{Hom}_{R}(K, D)\right) .
$$

Proof. We have an exact sequence

$$
0 \rightarrow \operatorname{Hom}_{R}(K, B) \stackrel{a}{\longrightarrow} \operatorname{Hom}_{R}(K, D) \stackrel{\beta}{\rightarrow} \operatorname{Hom}_{R}(K, D / B) .
$$

As we have remarked in the Introduction, all of these $R$-modules are torsion-free. Thus $Q$-rank $\left(\operatorname{Hom}_{R}(K, B)\right) \leq Q$-rank $\left(\operatorname{Hom}_{R}(K, D)\right)$. If these two $Q$-ranks are equal, then $\operatorname{Im} \beta$ is a torsion $R$-module, and hence $\operatorname{Im} \beta=0$. But then $\alpha$ is an isomorphism. Since $\operatorname{Hom}_{R}(K, B)=\operatorname{Hom}_{R}(K, b(B))$, we then have $b(B)=D$ by Corollary 1.3. Thus $B=D$, and this contradiction proves that $Q-\operatorname{rank}\left(\operatorname{Hom}_{R}(K, B)\right)<$ $Q$-rank $\left(\operatorname{Hom}_{R}(K, D)\right)$.

\section{Properties of $Q$-rings.}

Definition. We shall say that $R$ is a $Q$-ring if $\operatorname{Ext}_{R}^{1}(Q, R) \cong Q$.

Theorem 2.1. Suppose that there is an $R$-module $A$ such that $\operatorname{Hom}_{R}(A, R)=0$ and $\operatorname{Ext}_{R}^{1}(A, R) \simeq Q$. Then

(1) $R$ is a $Q$-ring,

(2) $\operatorname{Ext}_{R}^{1}(A / t(A), R) \simeq Q, \operatorname{Hom}_{R}(A / t(A), R)=0$, and $Q-\operatorname{rank}(A / t(A))=1$.

Proof. From the exact sequence: $0 \rightarrow \operatorname{Hom}_{R}(A / t(A), R) \rightarrow \operatorname{Hom}_{R}(A, R)$ we conclude that $\operatorname{Hom}_{R}(A / t(A), R)=0$. Now we have an exact sequence: 


$$
0 \rightarrow \operatorname{Ext}_{R}^{1}(A / t(A), R) \stackrel{a}{\longrightarrow} \operatorname{Ext}_{R}^{1}(A, R) \stackrel{\beta}{\longrightarrow} \operatorname{Ext}_{R}^{1}(t(A), R) .
$$

By [7, Theorem 2.1], $\operatorname{Ext}_{R}^{1}(t(A), R)$ is a cotorsion $R$-module, and by assumption $\operatorname{Ext}_{R}^{1}(A, R) \cong Q$. Thus $\beta=0$, and hence $a$ is an isomorphism. Therefore, $\operatorname{Ext}_{R}^{1}(A / t(A), R) \cong Q$. Thus without loss of generality we can assume that $A$ is torsion-free.

We shall next prove that $R$ is not complete in the $R$-topology. Suppose that $R$ is complete in the $R$-topology. Then $R \cong \operatorname{Hom}_{R}(K, K)$ by [7, Theorem 5.4], and hence

$$
\operatorname{Hom}_{R}\left(K \otimes_{R} A, K\right) \cong \operatorname{Hom}_{R}\left(A, \operatorname{Hom}_{R}(K, K)\right) \cong \operatorname{Hom}_{R}(A, R)=0 .
$$

Therefore, by [7, Theorem 2.1] we see that $\operatorname{Ext}_{R}^{1}\left(K \otimes_{R} A, K\right)$ is a cotorsion $R$ module. But $\operatorname{Ext}_{R}^{1}\left(K \otimes_{R} A, K\right) \cong \operatorname{Ext}_{R}^{2}\left(K \otimes_{R} A, R\right)$, and hence the latter module is also cotorsion. We have an exact sequence:

$$
\operatorname{Ext}_{R}^{1}\left(Q \otimes_{R} A, R\right) \rightarrow \operatorname{Ext}_{R}^{1}(A, R) \rightarrow \operatorname{Ext}_{R}^{2}\left(K \otimes_{R} A, R\right)
$$

Since $R$ is a cotorsion $R$-module, $\operatorname{Ext}_{R}^{1}\left(Q \otimes_{R} A, R\right)=0$. Thus $\operatorname{Ext}_{R}^{2}\left(K \otimes_{R} A, R\right)$ contains a copy of $\operatorname{Ext}_{R}^{1}(A, R) \cong Q$, and hence $\operatorname{Ext}_{R}^{2}\left(K \otimes_{R} A, R\right)$ is not b-reduced. This contradicts the fact that we have proved that $\operatorname{Ext}_{R}^{2}\left(K \otimes_{R} A, R\right)$ is a cotorsion $R$-module. The contradiction proves that $R$ is not complete in the $R$-topology.

Because $\operatorname{Hom}_{R}(A, R)=0$, we have an exact sequence:

$$
0 \rightarrow \operatorname{Ext}_{R}^{1}\left(K \otimes_{R} A, R\right) \rightarrow \operatorname{Ext}_{R}^{1}\left(Q \otimes_{R} A, R\right) \stackrel{\gamma}{\longrightarrow} \operatorname{Ext}_{R}^{1}(A, R) .
$$

Now $\operatorname{Ext}_{R}^{1}\left(K \otimes_{R} A, R\right)$ is a cotorsion $R$-module by [7, Theorem 2.1]; and since $\operatorname{Ext}_{R}^{1}(A, R)$ is torsion-free and $\operatorname{Ext}_{R}^{1}\left(Q \otimes_{R} A, R\right)$ is torsion-free and divisible, $\operatorname{Im} \gamma$ is also torsion-free and divisible. This implies that $\operatorname{Ext}_{R}^{1}\left(Q \otimes_{R} A, R\right) \cong$ $\operatorname{Ext}_{R}^{1}\left(K \otimes_{R} A, R\right) \oplus \operatorname{Im} \gamma$. But then $\operatorname{Ext}_{R}^{1}\left(K \otimes_{R} A, R\right)=0$, and hence $\gamma$ is a monomorphism. Since $R$ is not complete, $\operatorname{Ext}_{R}^{1}\left(Q \otimes_{R} A, R\right) \neq 0$. Because $\operatorname{Ext}_{R}^{1}(A, R) \cong Q$, we see that $\gamma$ is an isomorphism. Therefore $\operatorname{Ext}_{R}^{1}\left(Q \otimes_{R} A, R\right) \cong Q$.

If $\nu=Q$-rank $A$, then $Q \otimes_{R} A$ is a direct sum of $\nu$ copies of $Q$. Hence $\operatorname{Ext}_{R}^{1}\left(Q \otimes_{R} A, R\right)$ is a direct product of $\nu$ copies of $\operatorname{Ext}_{R}^{1}(Q, R)$. Because $\operatorname{Ext}_{R}^{1}\left(Q \otimes_{R} A, R\right) \cong Q$ we have $\nu=1$ and $\operatorname{Ext}_{R}^{1}(Q, R) \cong Q$. Therefore, $R$ is a $Q$-ring.

Theorem 2.2. The following statements are equivalent:

(1) $R$ is a $Q$-ring.

(2) $Q$-rank $H=2$.

(3) $\operatorname{Ext}_{R}^{1}(H, R) \cong K$.

(4) $\operatorname{Ext}_{R}^{1}(H, R)$ is a nonzero torsion $R$-module. 
Proof. By [7, Proposition 5.2] we have an exact sequence:

$$
0 \rightarrow R \rightarrow H \rightarrow \operatorname{Ext}_{R}^{1}(Q, R) \rightarrow 0 \text {. }
$$

Since $\operatorname{Ext}_{R}^{1}(Q, R)$ is torsion-free and divisible, this sequence makes the equivalence of (1) and (2) clearly apparent.

If we apply the functor $\operatorname{Hom}_{R}(\cdot, R)$ to exact sequence (a) we obtain an exact sequence:

$$
0 \rightarrow \operatorname{Hom}_{\mathrm{R}}(H, R) \rightarrow R \rightarrow \operatorname{Ext}_{R}^{1}\left(\operatorname{Ext}_{R}^{1}(Q, R), R\right) \rightarrow \operatorname{Ext}_{R}^{1}(H, R) \rightarrow 0 .
$$

By exact sequence (a) we see that $R \neq H$ if and only if $\operatorname{Ext}_{R}^{1}(Q, R) \neq 0$. Thus $R$ is not complete in any of the four cases that we are considering. Therefore, $\operatorname{Hom}_{R}(H, R)=0$ by [7, Proposition 5.11]. The equivalence of (1) and (4) now follows immediately from exact sequence (b).

If $R$ is a $Q$-ring, then exact sequence (b) becomes

$$
0 \rightarrow R \rightarrow Q \rightarrow \operatorname{Ext}_{R}^{1}(H, R) \rightarrow 0 .
$$

Thus $\operatorname{Ext}_{R}^{1}(H, R)$ is a torsion $R$-module, and hence $\operatorname{Tor}_{1}^{R}\left(K, \operatorname{Ext}_{R}^{1}(H, R)\right) \simeq$ $t\left(\operatorname{Ext}_{R}^{1}(H, R)\right)=\operatorname{Ext}_{R}^{1}(H, R)$. If we tensor exact sequence (c) with $K$ we obtain the isomorphism: $\operatorname{Tor}_{1}^{R}\left(K, \operatorname{Ext}_{R}^{1}(H, R)\right) \simeq K$. Hence we have proved that $\operatorname{Ext}_{R}^{1}(H, R) \cong K$, and this shows that $(1) \Rightarrow(3)$. It is trivial that (3) $\Rightarrow(4)$, and thus the proof of the theorem is complete.

Corollary 2.3. If $R$ is a $Q$-ring, then $H / R$ is isomorpbic to $Q$.

Proof. We have $H / R \cong \operatorname{Ext}_{R}^{1}(Q, R)$ by [7, Proposition 5.2], and thus $H / R \cong Q$.

Corollary 2.4. Suppose that $R$ is a $Q$-ring, and let $A$ be an $R$-module sucb that $R \subset A \varsubsetneqq Q$. Then the following statements are equivalent:

(1) Every $R$-bomomorphism from $R$ into $A$ can be lifted to an $R$-bomomorpbism from $H$ into $A$.

(2) $\operatorname{Hom}_{R}(H, A) \cong A$.

(3) $\operatorname{Hom}_{R}(H, A) \neq 0$.

(4) $A$ is an $H$-module.

(5) $A / R$ is not b-reduced

Proof. (1) $\Rightarrow$ (2). Define $\phi: \operatorname{Hom}_{R}(H, A) \rightarrow A$ by $\phi(f)=f(1)$ for all $f \epsilon$ $\operatorname{Hom}_{R}(H, A)$. Then (1) guarantees that $\phi$ is an epimorphism. If $f \in \operatorname{Ker} \phi$, then $f$ induces an $R$-homomorphism of $H / R$ into $A$. But $H / R$ is torsion-free and divisible, and $A$ is $b$-reduced. This implies that $f=0$ and hence $\phi$ is an $R$-isomorphism.

It is clear that (2) $\Rightarrow(4)$ and (4) $\Rightarrow(3)$.

$(3) \Rightarrow(1)$. We have an exact sequence:

$$
0 \rightarrow \operatorname{Hom}_{R}(H / R, A) \rightarrow \operatorname{Hom}_{R}(H, A) \stackrel{y}{\longrightarrow} \operatorname{Hom}_{R}(R, A) \stackrel{\delta}{\longrightarrow} \operatorname{Ext}_{R}^{1}(H / R, A) .
$$


Since $A$ is $b$-reduced and $H / R$ is torsion-free and divisible, we see that $\operatorname{Hom}_{R}(H / R, A)=0$. Because $Q$-rank $\operatorname{Hom}_{R}(R, A)=1$ and $\operatorname{Hom}_{R}(H, A) \neq 0$, we see that $\operatorname{Im} \delta$ is a torsion $R$-module. But $\operatorname{Ext}_{R}^{1}(H / R, A)$ is torsion-free, and thus $\delta=0$. Therefore, $\gamma$ is an isomorphism, and this establishes (1).

$(2) \Rightarrow(5)$. Suppose that $A / R$ is $b$-reduced. We have an exact sequence:

$$
0 \rightarrow \operatorname{Hom}_{R}(H / R, A / R) \rightarrow \operatorname{Hom}_{R}(H, A / R) \rightarrow A / R .
$$

Because $H / R$ is torsion-free and divisible, the first term of this sequence is zero, and thus $\operatorname{Hom}_{R}(H, A / R)$ is a torsion $R$-module. We have another exact sequence:

$$
0 \rightarrow \operatorname{Hom}_{R}(H, R) \rightarrow \operatorname{Hom}_{R}(H, A) \rightarrow \operatorname{Hom}_{R}(H, A / R) \rightarrow \operatorname{Ext}_{R}^{1}(H, R) .
$$

Because $R$ is not a cotorsion $R$-module, the first term of this sequence is zero by [7, Proposition 5.11]. Thus $\operatorname{Hom}_{R}(H, A / R)$ is not a torsion module, since it contains $\operatorname{Hom}_{R}(H, A) \cong A$. This contradiction shows that $A / R$ is not b-reduced.

$(5) \Rightarrow(3)$. Because $\operatorname{Hom}_{R}(H / R, A / R)$ is a nonzero torsion-free $R$-module, $\operatorname{Hom}_{R}(H, A / R)$ is not a torsion $R$-module by exact sequence (a). Since $\operatorname{Ext}_{R}^{l}(H, R)$ is a torsion $R$-module by Theorem 2.2, exact sequence (b) shows that $\operatorname{Hom}_{R}(H, A) \neq 0$.

Remarks. The equivalence of (1), (2), (3), and (4) in Corollary 2.4 is true for any integral domain, as we see from the proof. Furthermore $(2) \Rightarrow(5)$ requires only that $R$ not be a cotorsion $R$-module. Only in the proof of $(5) \Rightarrow(3)$ have we used the assumption that $R$ is a $Q$-ring.

Theorem 2.5. Let $S$ be an extension ring of $R$ in $Q$ such that $S / R$ is torsion of bounded order. Then we bave isomorphisms:

$$
\operatorname{Ext}_{R}^{1}(Q, R) \cong \operatorname{Ext}_{R}^{1}(Q, S) \cong \operatorname{Ext}_{S}^{1}(Q, S)
$$

Hence $R$ is a $Q$-ring if and only if $S$ is a $Q$-ring; and $R$ is complete in the $R$-topology if and only if $S$ is complete in the S-topology.

Proof. We have an exact sequence:

$$
\operatorname{Hom}_{R}(Q, S / R) \rightarrow \operatorname{Ext}_{R}^{1}(Q, R) \rightarrow \operatorname{Ext}_{R}^{1}(Q, S) \rightarrow \operatorname{Ext}_{R}^{1}(Q, S / R) .
$$

The extreme terms of this sequence are simultaneously torsion-free and torsion of bounded order, and hence these terms are zero. Thus we have $\operatorname{Ext}_{R}^{1}(Q, R) \cong$ $\operatorname{Ext}_{R}^{1}(Q, S)$. By [1, Chapter VI, Proposition 4.1.3] we have $\operatorname{Ext}_{R}^{1}(Q, S) \cong \operatorname{Ext}_{S}^{1}(Q, S)$.

Theorem 2.6. Suppose that $R$ is a $Q$-ring and let $B$ be an $R$-module sucb that $R \subset B \varsubsetneqq Q$. Then the following statements are equivalent:

(1) $\operatorname{Ext}_{R}^{1}(Q, B) \simeq Q$ and $B / R$ is b-reduced.

(2) $\widetilde{B} \cong H \otimes_{R} B$, where $\widetilde{B}$ is the completion of $B$ in the R-topology. 
Proof. (1) $\Rightarrow(2)$. Since $H / R$ is torsion-free and divisible, we see that $H$ is a flat $R \cdot$ module and that $H \otimes_{R} B / R \cong B / R$. Thus if we tensor the exact sequence $0 \rightarrow R \rightarrow B \rightarrow B / R \rightarrow 0$ with $H$ we obtain the exact sequence:

$$
0 \rightarrow H \rightarrow H \otimes_{R} B \rightarrow B / R \rightarrow 0 .
$$

Because $B / R$ is assumed to be $b$-reduced, we see from this exact sequence that $H \otimes_{R} B$ is an b-reduced $R$-module. By Corollary $2.3,(H / R) \otimes_{R} B \cong Q$; and thus if we tensor the exact sequence $0 \rightarrow R \rightarrow H \rightarrow H / R \rightarrow 0$ with $B$ we obtain the exact sequence:

$$
0 \rightarrow B \rightarrow H \otimes_{R} B \rightarrow Q \rightarrow 0 .
$$

Because $H \otimes_{R} B$ is $h$-reduced, we have $\operatorname{Hom}_{R}\left(Q, H \otimes_{R} B\right)=0$. Thus if we apply the functor $\operatorname{Hom}_{R}(Q, \cdot)$ to exact sequence (b) we obtain the exact sequence:

$$
0 \rightarrow \operatorname{Hom}_{R}(Q, Q) \rightarrow \operatorname{Ext}_{R}^{1}(Q, B) \rightarrow \operatorname{Ext}_{R}^{1}\left(Q, H \otimes_{R} B\right) \rightarrow 0 .
$$

But $\operatorname{Hom}_{R}(Q, Q) \cong Q$; and $\operatorname{Ext}_{R}^{1}(Q, B) \cong Q$ by assumption. Thus exact sequence (c) shows that $\operatorname{Ext}_{R}^{1}\left(Q, H \otimes_{R} B\right)=0$. Therefore, $H \otimes_{R} B$ is a cotorsion $R$-module. Therefore, $\operatorname{Ext}_{R}^{1}\left(K, H \otimes_{R} B\right) \simeq H \otimes_{R} B$ by [7, Corollary 2.3]. If we apply the functor $\operatorname{Hom}_{R}(K, \cdot)$ to exact sequence (b) we obtain the isomorphism: $\operatorname{Ext}_{R}^{1}(K, B)$ $\cong \operatorname{Ext}_{R}^{1}\left(K, H \otimes_{R} B\right)$. Thus we have $\operatorname{Ext}_{R}^{1}(K, B) \cong H \otimes_{R} B$. By [7, Theorem 6.10] we have $\widetilde{B} \cong \operatorname{Ext}_{R}^{1}(K, B)$, and hence $\widetilde{B} \cong H \otimes_{R} B$.

$(2) \Rightarrow$ (1). By [7, Theorem 6.10] $H \otimes_{R} B \cong \widetilde{B}$ is a cotorsion $R$-module. Hence if we apply the functor $\operatorname{Hom}_{R}(Q, \cdot)$ to exact sequence (b) we obtain the isomorphism $\operatorname{Hom}_{R}(Q, Q) \cong \operatorname{Ext}_{R}^{1}(Q, B)$. But $\operatorname{Hom}_{R}(Q, Q) \cong Q$, and thus $\operatorname{Ext}_{R}^{1}(Q, B) \cong Q$. From exact sequence (a) and [7, Lemma 1.1] we see that $B / R$ is an $h-r e d u c e d ~ R$-module.

Theorem 2.7. Suppose that $R$ is a $Q$-ring, and let $B$ be an $R$-module such that $R \subset B \subsetneq Q$ and $\widetilde{B} \cong H \otimes_{R} B$, where $\widetilde{B}$ is the completion of $B$ in the R-topology. Then $S=\operatorname{Hom}_{R}(B, B)$ is a Q-ring.

Proof. If we apply the functor $\operatorname{Hom}_{R}(\cdot, B)$ to the exact sequence $0 \rightarrow R \rightarrow B$ $\rightarrow B / R$ we obtain the exact sequence:

$$
0 \rightarrow S \rightarrow B \rightarrow \operatorname{Ext}_{R}^{1}(B / R, B)
$$

Thus we have an imbedding of $S$ into $B$ such that $B / S$ is isomorphic to a submodule of $\operatorname{Ext}_{R}^{1}(B / R, B)$. Because $\operatorname{Ext}_{R}^{1}(B / R, B)$ is a cotorsion $R$-module by [7, Theorem 2.1] we see that $B / S$ is an $h$-reduced $R$-module. We have an exact sequence:

$$
\operatorname{Hom}_{R}(Q, B / S) \rightarrow \operatorname{Ext}_{R}^{1}(Q, S) \rightarrow \operatorname{Ext}_{R}^{1}(Q, B) .
$$

Now $\operatorname{Hom}_{R}(Q, B / S)=0$ because $B / S$ is b-reduced; and $\operatorname{Ext}_{R}^{1}(Q, B) \cong Q$ by Theorem 2.6. Thus exact sequence (b) shows that $\operatorname{Ext}_{R}^{1}(Q, S)$ is equal to either 0 or $Q$. 
Suppose that $\operatorname{Ext}_{R}^{1}(Q, S)=0$. We have an exact sequence:

(c)

$$
\operatorname{Hom}_{R}(Q, S) \rightarrow \operatorname{Hom}_{R}(Q, S / R) \rightarrow \operatorname{Ext}_{R}^{1}(Q, R) \rightarrow \operatorname{Ext}_{R}^{1}(Q, S) .
$$

Since the extreme terms of this sequence are 0 , we have an isomorphism:

$\operatorname{Hom}_{R}(Q, S / R) \cong \operatorname{Ext}_{R}^{1}(Q, R)$; and thus $S / R$ is not $b$-reduced. However, $S / R$ is a submodule of $B / R$, and $B / R$ is $b$-reduced by Theorem 2.6. This contradiction shows that $\operatorname{Ext}_{R}^{1}(Q, S) \cong Q$.

By $\left[1\right.$, Chapter VI, Proposition 4.1.3] we have $\operatorname{Ext}_{R}^{1}(Q, S) \cong \operatorname{Ext}_{S}^{1}(Q, S)$. Therefore, $S$ is a $Q$-ring.

Remarks. We note in passing that if $S$ is the ring of Theorem 2.7, then we have verified the conditions of Theorem 2.6 for $S$; and thus $\tilde{S} \cong H \otimes_{R} S$, where $\tilde{S}$ is the completion of $S$ in the $R$-topology. The next theorem is thus a generalization of Theorem 2.7. It provides a very general way of finding new $Q$-rings from old ones.

Theorem 2.8. Suppose that $S$ is an integral domain containing $R$ and satisfying the following two conditions:

(1) The quotient field $L$ of $S$ is algebraic over $Q$.

(2) $\tilde{S} \cong H \otimes_{R} S$, where $\tilde{S}$ is the completion of $S$ in the R-topology.

Then $Q$-rank $\operatorname{Ext}_{R}^{1}(Q, R)=L$-rank $\operatorname{Ext}_{S}^{1}(L, S)$, and thus $R$ is a $Q$-ring if and only if $S$ is a $Q \cdot$ ring (for $L$ ).

Proof. By [7, Proposition 5.2] we have an exact sequence:

$$
\eta \rightarrow R \rightarrow H \rightarrow \operatorname{Ext}_{R}^{1}(Q, R) \rightarrow 0
$$

and thus we have an exact sequence:

$$
0 \rightarrow S \rightarrow H \otimes_{R} S \rightarrow \operatorname{Ext}_{R}^{1}(Q, R) \otimes_{R} S \rightarrow 0 .
$$

Because $H \otimes_{R} S$ is a complete $R$-module, it is a cotorsion $R$-module by [7, Corollary 6.4]. Thus we have an isomorphism:

$$
\operatorname{Hom}_{R}\left(Q, \operatorname{Ext}_{R}^{1}(Q, R) \bigotimes_{R} S\right) \simeq \operatorname{Ext}_{R}^{1}(Q, S) .
$$

But $\operatorname{Ext}_{R}^{1}(Q, S)=\operatorname{Ext}_{S}^{1}\left(Q \otimes_{R} S, S\right)$ by [1, Chapter VI, Proposition 4.1.3]; and clearly

$$
\operatorname{Hom}_{R}\left(Q, \operatorname{Ext}_{R}^{1}(Q, R) \otimes_{R} S\right) \simeq \operatorname{Ext}_{R}^{1}(Q, R) \otimes_{R} S .
$$

Thus we have an isomorphism:

$$
\operatorname{Ext}_{R}^{1}(Q, R) \otimes_{R} S \cong \operatorname{Ext}_{S}^{1}\left(Q \otimes_{R} S, S\right) .
$$

Let $Q$-rank $\operatorname{Ext}_{R}^{1}(Q, R)=c$, where $c$ is some cardinal number. Then $\operatorname{Ext}_{R}^{1}(Q, R)$ $\cong Q^{c}$, and hence $\operatorname{Ext}_{R}^{1}(Q, R) \otimes_{R} S \cong Q^{c} \otimes_{R} S \cong\left(Q \otimes_{R} S\right)^{c}$. By Corollary 1.6 
we have $Q \otimes_{R} S \cong L$, and thus $L^{c} \cong \operatorname{Ext}_{S}^{1}(L, S)$. It follows that $L$-rank $\operatorname{Ext}_{S}^{1}(L, S)=c$, and the theorem is proved.

Corollary 2.9. Let $S$ be an integral domain containing $R$, and suppose that $S$ is a subring of a finitely generated integral extension of $R$. Let $L$ be the quotient field of $S$. Then $R$ is a $Q$-ring if and only if $S$ is a $Q$-ring (for $L$ ).

Proof. $L$ is algebraic over $Q$, and hence by Theorem 2.8 it is sufficient to prove that $H \otimes_{R} S \cong \tilde{S}$, the completion of $S$ in the $R$-topology. Now there exists a finitely generated free $R$-module $F$ such that $S \subset F$ and $F / S$ is a torsion $R$-module (necessarily of bounded order). Because $H / R$ is torsion-free and divisible by [7, Proposition 5.2] we have $H \otimes_{R} F / S \cong F / S$. Thus we have an exact sequence:

$$
0 \rightarrow H \otimes_{R} S \rightarrow H \otimes_{R} F \rightarrow F / S \rightarrow 0
$$

Since $H \otimes_{R} F$ is a finite direct sum of copies of $H$ it is a cotorsion $R$-module; and of course $F / S$ is a cotorsion $R$-module. It follows that $H \otimes_{R} S$ is a cotorsion $R$-module. We have an exact sequence:

$$
0 \rightarrow S \rightarrow H \oplus_{R} S \rightarrow H / R \otimes_{R} S \rightarrow 0
$$

Because $H / R \otimes_{R} S$ is a torsion-free and divisible, it follows from [7, Theorem 6.10] that $H \otimes_{R} S \cong \tilde{S}$, the completion of $S$ in the $R$-topology. The corollary is now seen to be a special case of Theorem 2.8 .

Corollary 2.10. Suppose that $R$ is integrally closed and that $L$ is a finitedimensional and separable field extension of $Q$. Let $S$ be the integral closure of $R$ in $L$. Then $R$ is a Q-ring if and only if $S$ is a $Q$-ring (for $L$ ).

Proof. By induction on the number of generators of $L$ over $Q$ we may assume that $L=Q(s)$, where $s \in S$ and the monic minimal polynomial $f(x)$ of $s$ over $Q$ has coefficients in $R$. If $f^{\prime}(x)$ is the derivative of $f(x)$, then $f^{\prime}(s) S \subset R[s]$ by $\left[10\right.$, Theorem 10.18]. Because $L$ is separable over $Q$ we have $f^{\prime}(s) \neq 0$, and thus $S / R[s]$ is a torsion $R[s]$-module of bounded order. Hence by Theorem $2.5 S$ is a $Q$-ring (for $L$ ) if and only if $R[s]$ is a $Q$-ring (for $L$ ). The corollary is now an immediate consequence of Corollary 2.9.

3. The structure of $K$.

Lemma 3.1. Let $R$ be a $Q$-ring, and let $D$ be a proper, nonzero, b-divisible $R$-submodule of K. Then:

(1) $\operatorname{Hom}_{R}(K, D)$ is an ideal of $H$, and is a torsion-free cotorsion R-module of Q-rank 1.

(2) Every proper R-submodule of $D$ is b-reduced. 
Proof. $\operatorname{Hom}_{R}(K, D)$ is a torsion-free, cotorsion $R$-module by [7, Proposition 3.2]. Now $\operatorname{Hom}_{R}(K, D) \subset \operatorname{Hom}_{R}(K, K)=H$. Thus by Theorem 1.1, $\operatorname{Hom}_{R}(K, D)$ is an ideal of $H$. By Theorem 1.13, $Q$-rank $\operatorname{Hom}_{R}(K, D)<Q$-rank $H$; and by Theorem 2.2, $Q$-rank $H=2$. Since $\operatorname{Hom}_{A}(K, D) \neq 0$ by Theorem 1.2 , it follows that $Q$-rank $\operatorname{Hom}_{R}(K, D)=1$.

If $B$ is a proper, $b$-divisible submodule of $D$, then $Q$-rank $\operatorname{Hom}_{R}(K, B)<1$ by Theorem 1.13. Thus $\operatorname{Hom}_{R}(K, B)=0$, and hence $B=0$ by Corollary 1.3 .

Lemma 3.2. Let $R$ be a $Q$-ring and $f: K \rightarrow K$ an $R$-bomomorpbism sucb that Im $f \neq K$. Then Ker $f$ is not $b$-reduced.

Proof. Let $C=\operatorname{Ker} f$ and $D=\operatorname{Im} f$; then we have an exact sequence:

$$
0 \rightarrow \operatorname{Hom}_{R}(K, C) \rightarrow H \rightarrow \operatorname{Hom}_{R}(K, D) .
$$

By Lemma 3.1, $Q$-rank $\operatorname{Hom}_{R}(K, D)=1$; and by Theorem 2.2, $Q$-rank $H=2$. Thus $\operatorname{Hom}_{R}(K, C) \neq 0$, and hence $C$ is not $b$-reduced.

Theorem 3.3. Suppose that $R$ is a $Q$-ring and let $f: K \rightarrow K$ be a nonzero $R$-bomomorphism. Let $\operatorname{Ker} f=C / R$, where $C$ is an $R$-submodule of $Q$ containing R. Then

(1) If $\operatorname{Im} f \neq K$, then $C$ is complete in the R-topology.

(2) If $\operatorname{Im} f=K$, then $C$ is a projective $R$-module and can be generated by two elements.

Proof. By [7, Chapter III, p. 3], we have an exact sequence:

$$
0 \rightarrow C \rightarrow \operatorname{Ext}_{R}^{1}(K, C) \rightarrow \operatorname{Ext}_{R}^{1}(Q, C) \rightarrow 0 .
$$

Since $Q / C \cong K / \operatorname{Ker} f \cong \operatorname{Im} f$, and $\operatorname{Ext}_{R}^{1}(K, C) \cong \operatorname{Hom}_{R}(K, Q / C)$, it follows from this sequence that

$$
Q \text {-rank } \operatorname{Ext}_{R}^{1}(Q, C)=Q \text {-rank } \operatorname{Hom}_{R}(K, \operatorname{Im} f)-1 .
$$

Now if $\operatorname{Im} f \neq K$, then $Q$-rank $\operatorname{Hom}_{R}(K, \operatorname{Im} f)=1$ by Lemma 3.1 and hence $\operatorname{Ext}_{R}^{1}(Q, C)=0$ by $(*)$. Thus $C$ is a cotorsion $R$-module, and hence $C$ is complete in the $R$-topology by [7, Corollary 6.11].

On the other hand, suppose that $\operatorname{Im} f=K$. Then $Q$-rank $\operatorname{Hom}_{R}(K, \operatorname{Im} f)=2$ by Theorem 2.2 and hence $Q$-rank $\operatorname{Ext}_{R}^{1}(Q, C)=1$ by $(*)$. Therefore, $\operatorname{Ext}_{R}^{1}(Q, C) \cong Q$. Now $C / R$ is $b$-reduced. For if $C / R$ is not $b$-reduced, then by Theorem 1.2 there is a nonzero $R$-homomorphism $g: K \rightarrow K$ such that $g(K) \subset C / R$. Since $H$ is a commutative ring we have $0=f(g(K))=g(f(K))=g(K)$, and thus $g=0$. This contradiction shows that $C / R$ is b-reduced.

We have proved that the conditions of Theorem 2.6 are satisfied, and thus $\widetilde{C} \cong H \otimes_{R} C$, where $\widetilde{C}$ is the completion of $C$ in the $R$-topology. But $\widetilde{C} \cong$ $\operatorname{Hom}_{R}(K, Q / C)$ by [7, Proposition 3.2], and since $Q / C \cong K$, we have $H \otimes_{R} C \cong H$. 
It follows from [7, Proposition 9.1] that $C$ is a finitely generated projective $R$-module. Therefore $C / R$ is a torsion $R$-module of bounded order.

If we apply the functor $\operatorname{Hom}_{R}(K, \cdot)$ to the exact sequence $0 \rightarrow C / R \rightarrow K \stackrel{f}{\rightarrow}$ $K \rightarrow 0$, we obtain an exact sequence:

$$
\operatorname{Hom}_{R}(K, C / R) \rightarrow H \stackrel{g}{\rightarrow} H \rightarrow \operatorname{Ext}_{R}^{1}(K, C / R) \stackrel{\delta}{\rightarrow} \operatorname{Ext}_{R}^{1}(K, K) .
$$

Since $C / R$ has bounded order, we have $\operatorname{Hom}_{R}(K, C / R)=0$; and $\operatorname{Ext}_{R}^{1}(K, C / R)$ $\cong C / R$ by [7, Corollary 2.3]. By [7, Chapter III, p. 3] $\operatorname{Ext}_{R}^{1}(K, K) \cong \operatorname{Ext}_{R}^{1}(Q, K)$ is torsion-free. Thus $\delta=0$, and the preceding exact sequence becomes $0 \rightarrow H \stackrel{g}{\rightarrow}$ $H \rightarrow C / R \rightarrow 0$. By [7, Proposition 5.7], $\operatorname{Hom}_{R}(H, H)=H$, and thus $g \in H$. Hence we have $C / R \cong H / H g$.

Let $H g \cap R=I$, an ideal of $R$. Since $H g$ is the annihilator in $H$ of $H / H g$, it follows that $I$ is the annihilator of $C / R$ in $R$. Because $C / R$ has bounded order, $I$ is a nonzero ideal of $R$. It follows from [7, Propositions 7.8 and 5.10] that in this case $H / H g \cong R / I$. Therefore $C / R \cong R / I$, and from this isomorphism we see that $C$ can be generated by two elements.

Theorem 3.4. Suppose that $R$ is a $Q$-ring. Then $K$ bas at most two proper, nonzero, b-divisible submodules; and if two sucb modules do exist, then neither of them is contained in the other.

Proof. Suppose that $D$ is a proper, nonzero, $b$-divisible $R$-submodule of $K$. By Theorem 1.2 there is a nonzero $R$-homomorphism $f: K \rightarrow K$ such that $f(K) \subset D$. By Lemma 3.1, $f$ is an epimorphism. Suppose that $B$ is a proper, nonzero, $b$-divisible $R$-submodule of $K$ and $B \neq D$. By Theorem 1.1, $B$ is an $H$-submodule of $K$ and hence $f(B) \subset B \cap D$. By Lemma 3.1, $B \cap D$ is $b$-reduced, and thus $B \subset \operatorname{Ker} f$. If $A$ is another proper, nonzero, $b$-divisible $R$-submodule of $K$ such that $A \neq D$, then by the same reasoning we have $A \subset \operatorname{Ker} f$ as well. Therefore $A+B \subset \operatorname{Ker} f$, and since $A+B$ is $b$-divisible we have $B=A+B=A$ by Lemma 3.1. This proves that $K$ has at most two proper, nonzero, $b$-divisible $R$-submodules. If two such modules do exist, then by Lemma 3.1, neither one of them is contained in the other.

\section{The three kinds of $Q$-rings.}

Definition. By Theorem 3.4 there are only three possible kinds of $Q$-rings, which we shall label $Q_{0} \cdot, Q_{1} \cdot$, or $Q_{2}$-rings, according to whether or not $K$ has 0,1 , or 2 proper, nonzero, $b$-divisible $R$-submodules, respectively.

Definition. $R$ is called a closed domain if $J \cap R \neq 0$ for every nonzero ideal $J$ of $H$. Since $H / R$ is divisible, this is equivalent by Theorem 1.7 to saying that $H$ is a compatible ring extension of $R$. Thus by Theorem $1.9, H$ is an integral domain in this case, and the quotient field of $H$ is $H \otimes_{R} Q$.

If $R$ is a closed domain, then every proper submodule of $K$ is $b$-reduced. For if $D$ is a proper, nonzero, $b$-divisible $R$-submodule of $K$, then $J=\operatorname{Hom}_{R}(K, D)$ is 
a nonzero ideal of $H$ by Theorem 1.2. However, $J \cap R=0$ since every nonzero element of $R$ acts as an epimorphism on $K$. This contradiction proves that every proper submodule of $K$ is $b$-reduced.

We have proved [ 8 , Theorem 4.5] that for $R$ a quasi-local domain, then conversely if every proper $R$-submodule of $K$ is $b$-reduced, $R$ is a closed domain. The next theorem proves this same converse for $Q$-rings.

Theorem 4.1. A Q-ring is a closed domain if and only if it is a $Q_{0}$-ring. In this case $H$ is a compatible ring extension of $R$ and bence $H$ is an integral domain with quotient field $H \otimes_{R} Q$.

Proof. If a $Q$-ring $R$ is a closed domain, then by the remarks preceding this theorem $R$ is a $Q_{0}$-ring and $H$ is a compatible ring extension of $R$.

Conversely, assume that $R$ is a $Q_{0}$-ring. Suppose that $J$ is a nonzero ideal of $H$ such that $J \cap R=0$. By Zorn's lemma there is an ideal $P$ of $H$ that is maximal with respect to the properties that $P \supset J$ and $P \cap R=0$. Since $R$ is an integral domain, it is easy to see that $P$ is a prime ideal of $H$, and thus $H / P$ is a torsionfree $R$-module. Hence we have an exact sequence:

$$
0 \rightarrow K \otimes_{R} P \stackrel{a}{\rightarrow} K \otimes_{R} H \rightarrow K \otimes_{R} H / P \rightarrow 0 .
$$

Now $K \otimes_{R} P$ is not zero since $P$ is an $b$-reduced, torsion-free $R$-module; and thus Im $\alpha$ is a nonzero, $b$-divisible submodule of $K \otimes_{R} H$. But $K \otimes_{R} H \simeq K$ because $H / R$ is torsion-free and divisible. Since $R$ is a $Q_{0}$-ring it follows that $a$ is an isomorphism and therefore $K \otimes_{R} H / P=0$. This implies that $H / P$ is a nonzero torsion-free, divisible $R$-module, and hence the same is true of $\operatorname{Ext}_{R}^{1}(H / P, R)$. Since $\operatorname{Ext}_{R}^{1}(H, R)$ is a torsion $R$-module by Theorem 2.2, the exact sequence:

$$
\operatorname{Hom}_{R}(P, R) \rightarrow \operatorname{Ext}_{R}^{1}(H / P, R) \rightarrow \operatorname{Ext}_{R}^{1}(H, R)
$$

shows that $\operatorname{Hom}_{R}(P, R) \neq 0$. Because $P$ is an $H$-module, it follows that $\operatorname{Hom}_{R}(H, R)$ $\neq 0$. But since $R$ is not a cotorsion $R$-module, this contradicts [7, Proposition 5.11]. Thus we have proved that $R$ is a closed domain.

Theorem 4.2. Suppose that $R$ is a $Q_{0}$-ring and that inj $\operatorname{dim}_{R} R=1$. Let $A$ be a nonzero R-submodule of $Q$. Then $\operatorname{Hom}_{R}(A, R)=0$ if and only if $\operatorname{Ext}_{R}^{1}(A, R) \simeq Q$.

Proof. If $\operatorname{Hom}_{R}(A, R) \neq 0$, then $A$ is isomorphic to an ideal of $R$ and hence $\operatorname{Ext}_{R}^{1}(A, R)$ is a torsion $R$-module. Thus we cannot have $\operatorname{Ext}_{R}^{1}(A, R) \simeq Q$. Conversely, suppose that $\operatorname{Hom}_{R}(A, R)=0$. Then we have an exact sequence:

$$
0 \rightarrow \operatorname{Hom}_{R}(A, H) \rightarrow \operatorname{Hom}_{R}(A, Q) \rightarrow \operatorname{Ext}_{R}^{1}(A, R) \rightarrow \operatorname{Ext}_{R}^{1}(A, H) \rightarrow 0 .
$$

Since $\operatorname{Hom}_{R}(A, Q) \simeq Q$, it will be sufficient to prove that $\operatorname{Hom}_{R}(A, H)=0$ and $\operatorname{Ext}_{R}^{1}(A, H)=0$. 
Let $C=\operatorname{Hom}_{R}(A, H)$, and suppose that $C \neq 0$. Then by $(*)$ we can assume that $C$ is a nonzero submodule of $Q$. Without loss of generality we can assume that $R \subset C$. By [7, Theorem 2.1] $C$ is a cotorsion $R$-module. Since $R$ is not a cotorsion $R$-module, it follows from [7, Lemma 1.1] that $C / R$ is not $b$-reduced. This contradicts the fact that $R$ is a $Q_{0}$-ring, and thus $\operatorname{Hom}_{R}(A, H)=0$.

We have an exact sequence:

$$
\operatorname{Ext}_{R}^{1}(Q, H) \rightarrow \operatorname{Ext}_{R}^{1}(A, H) \rightarrow \operatorname{Ext}_{R}^{2}(Q / A, H) .
$$

The first term of this sequence is zero because $H$ is a cotorsion $R$-module, and the last term is zero because inj $\operatorname{dim}_{R} H=\operatorname{inj} \operatorname{dim}_{R} R=1$. Thus we have $\operatorname{Ext}_{R}^{1}(A, H)=0$, concluding the proof of the theorem.

Theorem 4.3. Suppose that $R$ is a $Q_{1}$-domain and let $A / R(R \subset A \subset Q)$ be the unique, proper, nonzero, $b$-divisible submodule of $K$. Let $P=\operatorname{Hom}_{R}(K, A / R)$. Then

(1) $P$ is the only minimal prime ideal of $H$. We have $Q$-rank $P=1$, and $P$ contains every ideal of $H$ that has $Q-r a n k 1 ; P \cap R=0$, and $P$ contains every ideal of $H$ with this property. Moreover, $K \otimes_{R} P$ is isomorphic to $A / R$.

(2) $A$ is a compatible ring extension of $R$ in $Q$; and $A$ is isomorpbic as a ring to $H / P$. $A$ is integral over $R$, and thus $R$ is not integrally closed.

Proof. (1) By Lemma 3.1, $P$ is an ideal of $H$ and $Q$-rank $P=1$. It is clear that if $f \in H=\operatorname{Hom}_{R}(K, K)$ then either $f \in P$ or $f$ is an epimorphism on $K$. It follows immediately from this that $P$ is a prime ideal of $H$ and that $P$ contains every zero divisor of $H$. If $f \in P$, then by Lemma 3.2, Ker $f$ is not $b$-reduced. Hence $A / R \subset \operatorname{Ker} f$, and this shows that $P^{2}=0$. Thus $P$ is the only minimal prime ideal of $H$, and $P$ is the set of zero divisors of $H$.

Let $I$ be an ideal of $H$ and let $g \in I$. If $g \notin P$, then $g$ is not a zero divisor of $H$ and hence $H g \cong H$. Since $Q$-rank $H=2$, we have $Q$-rank $I=2$. Thus if $Q$-rank $I=1$, we have $I \subset P$.

Since $P^{2}=0$, we have $P \cap R=0$. Let $J$ be a nonzero ideal of $H$ such that $J \cap R=0$. By Zorn's lemma we can assume that $J$ is maximal with respect to this property. It is then easy to see that $J$ is a prime ideal of $H$, and hence $H / J$ is a torsion-free $R$-module. Thus $Q$-rank $J=1$, and therefore $J \subset P$ by the preceding paragraph.

We have $K \otimes_{R} P \cong A / R$ by [7, Proposition 3.2]. By Corollary 1.8, $A$ is a compatible ring extension of $R$ in $Q$. By Corollary 2.4 the imbedding of $R$ into $A$ can be extended to an $R$-homomorphism $\phi: H \rightarrow A$ and $(\operatorname{Im} \phi) / R$ is not $b$-reduced. Since $A / R$ is the only $b$-divisible submodule of $K$, we have $\operatorname{Im} \phi=A$. Let $b$ be a fixed element of $H$ and define an $R$-homomorphism $\lambda: H \rightarrow$. $A$ by $\lambda(g)=\phi(g b)-$ $\phi(g) \phi(b)$ for all $g \in H$. Then $R \subset \operatorname{Ker} \lambda$ and hence $\lambda$ induces an $R$-homomorphism of $H / R$ into $A$. Since $H / R$ is torsion-free and divisible and $A$ is $b$-reduced, we 
see that $\lambda=0$. Therefore, $\phi$ is a ring homomorphism. Thus $\operatorname{Ker} \phi$ is a prime ideal of $H$ and $\operatorname{Ker} \phi \cap R=0$. It follows from the preceding paragraph that $\operatorname{Ker} \phi=P$, and hence $H / P$ and $A$ are isomorphic rings.

Let $V$ be a valuation ring in $Q$ containing $R$. Suppose that $A \not \subset V$; then $V / R$ is an $b$-reduced $R$-module. Hence by Theorem 1.10 we have a ring imbedding of $H$ into $H(V)$, the completion of $V$. But $H(V)$ is an integral domain by [7, Proposition 8.8], and $H$ is not an integral domain as we have seen. This contradiction shows that $A \subset V$, and from this it follows that $A$ is integral over $R$.

Definition. We will say that $R$ is a special $Q_{2}$-ring if there are two $R$-submodules $A_{1}$ and $A_{2}$ of $Q$ such that $A_{1}+A_{2}=Q, A_{1} \cap A_{2}=R$, and $A_{1}$ and $A_{2}$ are complete in the $R$-topology.

Lemma 4.4. A special $Q_{2}$-ring is a $Q_{2}$-ring.

Proof. Assume that $R$ is a special $Q_{2}$-ring. Then we have an exact sequence: $0 \rightarrow R \rightarrow A_{1} \oplus A_{2} \rightarrow Q \rightarrow 0$. Thus we have a derived exact sequence:

$$
\operatorname{Hom}_{R}\left(Q, A_{1} \oplus A_{2}\right) \rightarrow \operatorname{Hom}_{R}(Q, Q) \rightarrow \operatorname{Ext}_{R}^{1}(Q, R) \rightarrow \operatorname{Ext}_{R}^{1}\left(Q, A_{1} \oplus A_{2}\right) .
$$

Since $A_{1} \oplus A_{2}$ is a cotorsion $R$-module by $[7$, Corollary 6.11], the extreme terms of this sequence are zero, and thus $\operatorname{Ext}_{R}^{1}(Q, R) \simeq \operatorname{Hom}_{R}(Q, Q) \simeq Q$. Therefore, $R$ is a $Q$-ring. We have $A_{1} / R \cong Q / A_{2}$ and $A_{2} / R \cong Q / A_{1}$, and thus $A_{1} / R$ and $A_{2} / R$ are distinct, proper, nonzero, $b$-divisible submodules of $K$. Therefore, $R$ is a $Q_{2}$-ring.

Theorem 4.5. Suppose that $R$ is a $Q_{2}$-ring and let $A_{1} / R$ and $A_{2} / R$ be the two distinct, proper, nonzero, $b$-divisible $R$-submodules of $K$. Let $P_{i}=$ $\operatorname{Hom}_{R}\left(K, A_{i} / R\right)$ for $i=1,2$. Then

(1) $P_{1}$ and $P_{2}$ are distinct prime ideals of $H, P_{1} \cap P_{2}=0$, and $P_{1}$ and $P_{2}$ are the only minimal prime ideals of $H$. The set of zero divisors of $H$ is equal to $P_{1} \cup P_{2}$. We have $Q$-rank $P_{i}=1$ for $i=1,2$ and every ideal of $H$ of $Q$-rank 1 is contained in either $P_{1}$ or $P_{2} ; P_{i} \cap R=0$, and every ideal of $H$ with this property is contained in $P_{1}$ or $P_{2}$. Moreover $K \otimes_{R} P_{i} \simeq A_{i} / R$ for $i=1,2$.

(2) $A_{1}$ and $A_{2}$ are compatible ring extensions of $R . A_{i}$ is complete in the $A_{i}$ topology and $A_{i}$ is isomorpbic as a ring to $H / P_{i}$ for $i=1,2$. We bave $A_{1}+$ $A_{2}=Q, A_{1} \cap A_{2}$ is integral over $R$, and $\left(A_{1} \cap A_{2}\right) / R$ is a torsion $R$-module of bounded order. $A_{1} \cap A_{2}$ is a special $Q_{2}$-ring. The integral closure of $R$ in $Q$ is equal to the intersection of the integral closures of $A_{1}$ and $A_{2}$ in $Q$.

Proof. $A_{1} / R+A_{2} / R$ is an $b$-divisible submodule of $K$ properly containing $A_{1} / R$. Thus by Lemma 3.1, $A_{1} / R+A_{2} / R=K$ and hence $A_{1}+A_{2}=Q$. It also follows from Lemma 3.1 that $A_{1} / R \cap A_{2} / R$ is an $b$-reduced $R$-module. Therefore, $P_{1} \cap P_{2}=\operatorname{Hom}_{R}\left(K, A_{1} / R \cap A_{2} / R\right)=0$. By Lemma 3.1, $P_{1}$ and $P_{2}$ are ideals of $H$ and $Q$-rank $P_{i}=1$ for $i=1,2$. 
It is clear that an element of $H$ is either an epimorphism on $K$ or an element of $P_{1} \cup P_{2}$. Thus every zero divisor of $H$ is in $P_{1} \cup P_{2}$. On the other hand the fact that $P_{1} P_{2}=0$ shows that every element of $P_{1} \cup P_{2}$ is a zero divisor of $H$.

We shall show next that $P_{1}$ is a prime ideal of $H$. Suppose that $f$ and $g$ are elements of $H-P_{1}$ such that $f g \in P_{1}$. Then neither $f$ nor $g$ is an epimorphism on $K$ and hence both $f$ and $g$ are in $P_{2}$. Thus $f g \in P_{1} \cap P_{2}=0$. Since $g(K)=$ $A_{2} / R$, we have $A_{2} / R \subset \operatorname{Ker} f$. Now $f\left(A_{1} / R\right) \subset A_{1} / R$ by Theorem 1.1 , and hence $f\left(A_{1} / R\right) \subset A_{1} / R \cap A_{2} / R$. Since $A_{1} / R \cap A_{2} / R$ is an $b$-reduced $R$-module, we have $A_{1} / R \subset \operatorname{Ker} f$. Therefore, $K=A_{1} / R+A_{2} / R \subset \operatorname{Ker} f$, and thus $f=0$. This contradiction shows that $P_{1}$ is a prime ideal of $H$. In similar fashion we prove that $P_{2}$ is a prime ideal of $H$. Since $P_{1} P_{2}=0$, it is clear that $P_{1}$ and $P_{2}$ are the only minimal prime ideals of $H$.

Suppose that an ideal $J$ of $H$ contains a nonzero divisor $g$. Then $\mathrm{Hg} \simeq \mathrm{H}$, and since $Q$-rank $H=2$ by Theorem 2.2 , we have $Q$-rank $J=2$. Thus if $J$ is an ideal of $H$ such that $Q$-rank $J=1$, then every element of $J$ is a zero divisor of $H$, and thus $J \subset P_{1} \cup P_{2}$. Since $P_{1}$ and $P_{2}$ are prime ideals, we have $J \subset P_{1}$ or $J \subset P_{2}$.

Since every element of $R$ acts on an epimorphism on $K$, we have $P_{i} \cap R=0$ for $i=1,2$. Let $J$ be an ideal of $H$ such that $J \cap R=0$. By Zorn's lemma we can assume that $J$ is maximal with respect to this property. It is then easy to see that $J$ is a prime ideal of $H$, and hence $H / J$ is a torsion-free $R$-module. Therefore $Q$-rank $J=1$, and hence $J \subset P_{1}$ or $J \subset P_{2}$ by the preceding paragraph.

By [7, Proposition 3.2] we have $K \otimes_{R} P_{i} \simeq A_{i} / R$ for $i=1,2 . A_{1}$ and $A_{2}$ are compatible ring extensions of $R$ by Corollary 1.8. Because $P_{1}+P_{2}$ is an ideal of $H$ not contained in either $P_{1}$ or $P_{2}$, we see by what we have already proved that there is a nonzero element $r$ of $R$ in $P_{1}+P_{2}$. Thus $r=f+g$, where $f \in P_{1}$ and $g \in P_{2}$. By Theorem 1.1, $f\left(A_{2} / R\right) \subset A_{1} / R \cap A_{2} / R$, and the latter module is $b$-reduced. Thus $f\left(A_{2} / R\right)=0$, and similarly $g\left(A_{1} / R\right)=0$. Hence we have $r\left(A_{1} \cap A_{2}\right) / R=0$, showing that $\left(A_{1} \cap A_{2}\right) / R$ is a torsion $R$-module of bounded order.

Let $S=A_{1} \cap A_{2}$; since $A_{1}+A_{2}=Q$, we have an exact sequence: $0 \rightarrow S \rightarrow$ $A_{1} \oplus A_{2} \rightarrow Q \rightarrow 0$. Thus we have an exact sequence:

$$
0 \rightarrow \operatorname{Hom}_{R}(Q, Q) \rightarrow \operatorname{Ext}_{R}^{1}(Q, S) \rightarrow \operatorname{Ext}_{R}^{1}\left(Q, A_{1} \oplus A_{2}\right) \rightarrow 0 .
$$

Now $\operatorname{Hom}_{R}(Q, Q) \cong Q$; and since $S / R$ is a torsion $R$-module of bounded order, we have $\operatorname{Ext}_{R}^{1}(Q, S) \cong Q$ by Theorem 2.5. Therefore, the preceding exact sequence shows that $\operatorname{Ext}_{R}^{1}\left(Q, A_{1} \oplus A_{2}\right)=0$. Thus $A_{1}$ and $A_{2}$ are complete in the $R$-topology by [7, Corollary 6.11]; and hence $A_{i}$ is complete in the $A_{i}$-topology for $i=1,2$ by Theorem 1.5 .

Since $H\left(A_{i}\right)=A_{i}$, we have an exact sequence by Theorem 1.10:

$$
0 \rightarrow H / P_{i} \stackrel{\phi_{i}}{\rightarrow} A_{i} \rightarrow \operatorname{Ext}_{R}^{1}\left(K, A_{i} / R\right)
$$


where $\phi_{i}$ is a ring homomorphism. By [7, Chapter III, p. 3], $\operatorname{Ext}_{R}^{1}\left(K, A_{i} / R\right)$ is torsion-free and divisible. But since $Q$-rank $A_{i}=1, A_{i} / \operatorname{Im} \phi_{i}$ is a torsion $R$-module. Therefore, $\phi$ is an isomorphism and $A_{i}$ is isomorphic to $H / P_{i}$ as a ring.

Let $V$ be a valuation ring such that $R \subset V \subset Q$. If $V / R$ is $b$-reduced, then by Theorem 1.10 we have $H \subset H(V)$, where $H(V)$ is the completion of $V$. But $H(V)$ is an integral domain by [7, Proposition 8.8] and $H$ is not an integral domain. This contradiction shows that $V / R$ is not $b$-reduced; and hence $A_{1} / R \subset V / R$ or $A_{2} / R$ $C V / R$. Thus $S \subset V$, and therefore, $S$ is integral over $R$. Furthermore, the integral closure of $R$ in $Q$ is equal to the intersection of the integral closures of $A_{1}$ and $A_{2}$ in $Q$.

Since $A_{i}$ is an $S$-module, $A_{i}$ is complete in the $S$-topology by Theorem 1.5. Therefore, by Lemma $4.4, S$ is a special $Q_{2}$-ring.

Corollary 4.6. Suppose that $R$ is a $Q$-ring, and let $P$ be a minimal prime ideal of $H$. Then $H / P$ is a compatible ring extension of $R$.

Proof. The proof is an immediate consequence of Theorems 4.1, 4.3 and 4.5.

Definition. If $S$ is a ring and $A$ is a free $S$-module on one generator $x$, then $S \oplus A$ can be made into a ring extension of $S$ by using the addition of $S \oplus A$ and defining multiplication in $S \oplus A$ by

$$
\left(s_{1}+t_{1} x\right)\left(s_{2}+t_{2} x\right)=s_{1} s_{2}+\left(s_{1} t_{2}+t_{1} s_{2}\right) x \quad\left(s_{1}, s_{2}, t_{1}, t_{2} \in S\right) .
$$

This equivalent to putting the zero multiplication on $A$ and defining the product of elements of $S$ and $A$ by the $S$-module structure of $A$. Such an extension is called the ring of dual numbers over $S$ [1, Chapter IV, \$2].

Corollary 4.7. Suppose that $R$ is a $Q$-ring and let $\Lambda$ be the full ring of quotients of $H$. Then $\Lambda \cong H \otimes_{R} Q$ is a two-dimensional commutative $Q$-algebra, and

(1) If $R$ is a $Q_{0}$-ring, $\Lambda$ is a quadratic field extension of $Q$.

(2) If $R$ is a $Q_{1}$-ring, $\Lambda$ is the ring of dual numbers over $Q$.

(3) If $R$ is a $Q_{2}$-ring, $\Lambda$ is the ring direct sum of two copies of the field $Q$.

Proof. (1) If $R$ is a $Q_{0}$-ring, then $H$ is an integral domain by Theorem 4.1. Then $\Lambda$ is the quotient field of $H$. Because $Q-\operatorname{rank} H=2$ by Theorem $2.2, \Lambda$ is a quadratic field extension of $Q$. We have $\Lambda \cong H \otimes_{R} Q$ by Corollary 1.6.

(2) Assume that $R$ is a $Q_{1}$-ring. We shall apply Theorem 4.3. Let $P$ be the unique minimal prime ideal of $H$. Then $P$ is the set of zero divisors of $H$, and thus $H_{P}=\Lambda$. If $f$ is a nonzero divisor in $H$, then $H f \cap R \neq 0$. From this it follows that $\Lambda \cong H \otimes_{R} Q$. Hence $\Lambda$ is a two-dimensional commutative $Q$-algebra.

The maximal ideal of $H_{P}$ is $P H_{P}$, and $H_{P} / P H_{P}$ is isomorphic to the quotient field of $H / P$. Since $H / P$ is isomorphic to a subring of $Q$ containing $R$, we see that $H_{P} / P H_{P} \cong Q$. Now $\left(P H_{P}\right)^{2}=P^{2} H_{P}=0$, and thus $P H_{P}$ is an $H_{P} / P H_{P}$-module; 
that is, $P H_{P}$ is a $Q$-submodule of $\Lambda$. Because $Q$-rank $\left(H \otimes_{R} Q\right)=2$, we have $P H_{P} \cong Q$ as a $Q$-module. Therefore $\Lambda$ is the ring of dual numbers over $Q$.

(3) Assume that $R$ is a $Q_{2}$-ring. We shall apply Theorem 4.5. Let $P_{1}$ and $P_{2}$ be the two distinct minimal prime ideals of $H$. Then $P_{1} \cup P_{2}$ is the set of zero divisors in $H$, and hence we have $\Lambda=H_{P_{1} \cup P_{2}}$. If $f$ is a nonzero divisor of $H$, then $H f \cap R \neq 0$. From this it follows that $\Lambda \cong H \otimes_{R} Q$. Hence $\Lambda$ is a two-dimensional commutative $Q$-algebra.

Now $\Lambda$ has two distinct maximal ideals $P_{1} \Lambda$ and $P_{2} \Lambda$, and thus $\Lambda=P_{1} \Lambda+P_{2} \Lambda$. We have $P_{1} \Lambda \cap P_{2} \Lambda=\left(P_{1} \cap P_{2}\right) \Lambda=0$, and therefore, $\Lambda$ is isomorphic as a ring to $\Lambda / P_{1} \Lambda \oplus \Lambda / P_{2} W_{0}$ It is a standard result of localizations that $\Lambda / P_{i} \Lambda \cong H_{P_{i}} / P_{i} H_{P_{i}}$. As in (2) we see that $H_{P_{i}} / P_{i} H_{P_{i}}$ and $Q$ are isomorphic rings. Therefore, $\Lambda$ is isomorphic as a ring to $Q \oplus Q$.

Remarks. It is easily seen that there are only three possible kinds of twodimensional commutative $Q$-algebras-namely a quadratic field extension of $Q$, the ring of dual numbers over $Q$, and the ring direct sum of two copies of $Q$. We see from Corollary 4.7 that if $R$ is a $Q$-ring, then the full ring of quotients of $H$ can be any one of these, depending upon whether $R$ is a $Q_{0^{-}}, Q_{1}^{-}$, or $Q_{2}$-ring. This perhaps serves to explain why there are precisely three kinds of $Q$-rings.

Theorem 4.8. Assume that $R$ is a $Q_{0}$-ring. Then there exists an element $x$ of $H-R$ that is integral over $R$. If the quotient field of $H$ is separable over $Q$, then $R[x]$ is a $Q_{2}$-ring; otherwise $R[x]$ is a $Q_{1}$-ring, and $R$ bas characteristic 2 .

Proof. $H$ is an integral domain by Theorem 4.1; let $L$ be its quotient field. Since $L$ has dimension 2 over $Q$, it is easy to see that there exists an element $x$ of $H-R$ that is integral over $R$. Let $S=R[x]$; then $L$ is also the quotient field of $S$. By Corollary $2.9, S$ is a $Q$-ring for $L$. The canonical epimorphism: $H / R \rightarrow H / S \rightarrow 0$ shows that $H / S$ is an $b$-divisible $R$-module. Hence by Theorem 1.5, $H / S$ is an $b$-divisible $S$-module. Since $H \neq L, H / S$ is a proper submodule of $L / S$. $H / S$ is not zero, for $H$ can not be a finitely generated $R$-module by [7, Proposition 5.11]. Thus $S$ is not a $Q_{0}$-ring.

Assume that $S$ is a $Q_{1}$-ring, and let $\sigma$ be an automorphism of $L$ over $Q$. Since $H / S$ is the unique, proper, nonzero, $b$-divisible $S$-submodule of $L / S, H$ is integral over $S$ by Theorem 4.3. Thus $H+\sigma(H)$ is contained in the integral closure $\Gamma$ of $R$ in $L$. Since $Q \otimes_{R} S=L$ by Corollary 1.6 we have by [1, Chapter VI, Proposition 4.1.3] that $\operatorname{Hom}_{R}(Q, \Gamma) \cong \operatorname{Hom}_{S}\left(Q \otimes_{R} S, \Gamma\right) \cong \operatorname{Hom}_{S}(L, \Gamma)=0$. Thus $\Gamma$ is an $b$-reduced $R$-module. Hence $H+\sigma(H)$ is also an $b$-reduced $R$-module. Because $H$ and $\sigma(H)$ are cotorsion $R$-modules, it follows from the exact sequence:

$$
0 \rightarrow H \cap \sigma(H) \rightarrow H \oplus \sigma(H) \rightarrow H+\sigma(H) \rightarrow 0
$$

that $H \cap \sigma(H)$ is a cotorsion $R$-module containing $R$. But there are no cotorsion 
$R$-modules properly between $R$ and $H$ by [7, Corollary 2.6]. Hence we have $H=$ $H \cap \sigma(H)$, and thus $H \subset \sigma(H)$. Since $\sigma^{2}$ is the identity on $L$, we have $H=\sigma(H)$.

Let $\eta$ be the restriction of $\sigma$ to $H$; then $\eta$ is an $R$-endomorphism of $H$. By [7, Proposition 5.7], $\eta$ is also an $H$-homomorphism. Thus if $b \in H$, we have $\eta(b)=$ $b \eta(1)=b \cdot 1=b$, and thus $\sigma$ leaves every element of $H$ fixed. Therefore, $\sigma$ is the identity on $L$. We have proved that the Galois group of $L$ over $Q$ contains only the identity mapping. Thus $L$ is not separable over $Q$. Because $[L: Q]=2, R$ has characteristic 2. Hence we have shown that if $L$ is separable over $Q$, then $S$ is a $Q_{2}$-ring.

Conversely, assume that $S$ is a $Q_{2}$-ring. Then $H / S$ is one of the two proper, nonzero, $b$-divisible $S$-submodules of $L / S$. Denote the other one by $A / S$, where $S \subsetneq A \varsubsetneqq L$. By Theorem 4.5, $A$ is complete in the $A$-topology and $A+H=L$. By [7, Corollary 6.11] and Theorem 1.5 we see that $A$ is a cotorsion $R$-module. By [7, Corollary '5.8] the imbedding of $R$ into $A$ can be extended to a ring homomorphism $\nu$ of $H$ into $A$.

Now $\operatorname{Ker} \nu$ is an ideal of $H$ and $\operatorname{Ker} \nu \cap R=0$. Since $R$ is a closed domain by Theorem 4.1, we see that $\operatorname{Ker} \nu=0$. Thus $\nu$ is a ring monomorphism of $H$ into $A$. We can extend $\nu$ to an automorphism $r$ of $L$ over $Q$. Since $A+H=L$ and $r(H) \subset A$ we see that $r$ is not the identity mapping. Therefore the Galois group of $L$ over $Q$ has two elements, proving that $L$ is separable over $Q$. Thus if $L$ is not separable over $Q$, then $S$ is a $Q_{1}$-ring.

Corollary 4.9. Suppose that $R$ is a $Q_{0}$-ring, and that $F$ is a finite-dimensional field extension of $Q$ that does not contain a copy of the quotient field $L$ of $H$ (for example if $[F: Q]$ is not divisible by 2). Let $x$ be an element of $F$ that is integral over $R$. Then $R[x]$ is a $Q_{0}$-ring.

Proof. $R[x]$ is a $Q$-ring by Corollary 2.9. Suppose that $R[x]=B$ is not a $Q_{0^{-}}$ ring. Then by Theorem 4.3 or Theorem 4.5 there is a ring $A$ such that $R[x] \lessgtr A \lessgtr F$ and $A$ is a ring homomorphic image of $H(B)$, the completion of $B$ in the $B$-topology. $H(B)$ is complete in the R-topology by Theorem 1.5 , and hence $H(B)$ is a cotorsion $R$-module by [7, Proposition 6.3]. Thus by [7, Corollary 5.8] the imbedding of $R$ into $B$ can be extended to a ring homomorphism of $H$ into $H(B)$. The composite $H \rightarrow H(B) \rightarrow A \rightarrow 0$ is a ring homomorphism of $H$ into $A$ extending the imbedding of $R$ into $A$. If $P$ is the kernel of this map, then $P \cap R=0$. Since $R$ is a closed domain by Theorem 4.1, we have a ring monomorphism of $H$ into $A$. Thus $F$ contains a copy of the quotient field of $H$, contradicting our assumption. Therefore, $R$ is a $Q_{0}$-ring.

Corollary 4.10. Let $R$ be an integrally closed $Q_{0}$-ring. If the quotient field $L$ of $H$ is separable over $Q$, then the integral closure $\Gamma$ of $R$ in $L$ is a special $Q_{2}$-ring. 
Proof. By Corollary 2.10, $\Gamma$ is a $Q$-ring (for $L$ ). It is clear from Theorem 4.8 that $\Gamma$ is not a $Q_{0}$-ring. By Theorem 4.3 , a $Q_{1}$-ring is not integrally closed, and thus $\Gamma$ is a $Q_{2}$-ring. By Theorem 4.5 an integrally closed $Q_{2}$-ring is a special $Q_{2}$-ring.

Corollary 4.11. Let $R$ be a $Q_{0}$-ring; then the quotient field $L$ of $H$ is inseparable over $Q$ if and only if $H$ is integral over $R$. In this case $R$ bas characteristic 2.

Proof. If $L$ is inseparable over $Q$, and $x \in H$, then $x^{2} \in H \cap Q=R$; and hence $H$ is integral over $R$. Conversely, suppose that $H$ is integral over $R$. Let $\sigma$ be an automorphism of $L$ over $Q$. Then $H+\sigma(H)$ is integral over $R$, and as in the proof of Theorem 4.8 we see that $\sigma$ is the identity, and thus $L$ is inseparable over $Q$.

\section{Special $Q_{2}$-rings.}

Theorem 5.1. An integral domain $R$ is a $Q_{2}$-ring if and only if there exists a special $Q_{2}$-ring $S$ between $R$ and $Q$ such that $S / R$ is a torsion $R$-module of bounded order.

Proof. If $R$ is a $Q_{2}$-ring, then the existence of $S$ has been proved in Theorem 4.5. Conversely, suppose that $S$ with the desired properties exists. Then by Theorem 2.5, $R$ is a $Q$-ring. By definition, there exist two $S$-modules $A_{1}$ and $A_{2}$ such that $A_{1}+A_{2}=Q, A_{1} \cap A_{2}=S$, and $A_{1}$ and $A_{2}$ are complete in the $S$-topology. By Theorem 1.5, $A_{1}$ and $A_{2}$ are complete in the $R$-topology. Since $R$ is not complete in the $R$-topology, $A_{1} / R$ and $A_{2} / R$ are not $b$-reduced $R$-modules. Because $A_{1} / R$ $\cap A_{2} / R=S / R$ is a torsion $R$-module of bounded order, we see that $K$ has at least two proper nonzero, $b$-divisible submodules. Thus $R$ is a $Q_{2}$-ring.

Theorem 5.2. Suppose that $R$ is a special $Q_{2}$-ring and let $A_{1} / R$ and $A_{2} / R$ be the two proper, nonzero, b-divisible submodules of $K$. For $i=1,2$ let $\mathcal{C}_{i}$ be the set of all maximal ideals $M$ of $R$ such that $A_{i} \subset R_{M}$. Then

(1) $\mathcal{C}_{1} \cup \mathcal{C}_{2}$ is the set of all maximal ideals of $R$ and $\mathcal{C}_{1} \cap \mathcal{C}_{2}$ is the empty set.

(2) $A_{i}=\bigcap R_{M},\left(M \in \mathcal{C}_{i}\right)$ for $i=1,2$.

(3) If $M \in \mathcal{C}_{1}$ and $N \in \mathcal{C}_{2}$, then $R_{M} \otimes_{R} R_{N}=Q$ and thus $M \cap N$ does not contain any nonzero prime ideals of $R$.

Proof. (1) We have $A_{1}+A_{2}=Q$ and $A_{1} \cap A_{2}=R$. Hence, if $M$ is a maximal ideal of $R$, then $Q=\left(A_{1}\right)_{M}+\left(A_{2}\right)_{M}$ and $R_{M}=\left(A_{1}\right)_{M} \cap\left(A_{2}\right)_{M}$. Thus $Q / R_{M}=$ $\left(A_{1}\right)_{M} / R_{M} \oplus\left(A_{2}\right)_{M} / R_{M}$. However $R_{M}$ is a quasi-local ring and thus $Q / R_{M}$ is an indecomposable $R_{M}$-module by [8, Corollary 4.2]. Thus we can assume that $\left(A_{1}\right)_{M}=R_{M}$ and $\left(A_{2}\right)_{M}=Q$. Therefore, $M \in \mathcal{C}_{1}$ and $M \notin \mathcal{C}_{2}$ and thus $\mathcal{C}_{1} \cap \mathcal{C}_{2}$ 
is the empty set and $\mathcal{C}_{1} \cup \mathcal{C}_{2}$ is the set of all maximal ideals of $R$.

(2) Now $A_{2}=\bigcap_{M}\left(A_{2}\right)_{M}$, where $M$ ranges over all maximal ideals of $R$, and $\left(A_{2}\right)_{M}=Q$ for $M \in \mathcal{C}_{1}$. Therefore $A_{2}=\bigcap_{N}\left(A_{2}\right)_{N}$, where $N$ ranges over all maximal ideals in $\mathcal{C}_{2}$. We have seen in (1) that $\left(A_{2}\right)_{N}=R_{N}$ for all $N \in \mathcal{C}_{2}$. Thus we have $A_{2}=\bigcap_{N} R_{N}\left(N \in \mathcal{C}_{2}\right)$. Similarly, we have $A_{1}=\bigcap R_{M}\left(M \in \mathcal{C}_{1}\right)$.

(3) If $M \in \mathcal{C}_{1}$ and $N \in \mathcal{C}_{2}$, then $Q=\left(A_{2}\right)_{M}$ and $R_{N}=\left(A_{2}\right)_{N}$. Therefore $R_{M} \otimes R_{N} \cong Q$, and hence by [7, Lemma 8.1] $M \cap N$ does not contain any nonzero prime ideals of $R$.

Theorem 5.3. Suppose that $R$ is a $Q_{2}$-ring and let $A_{1} / R$ and $A_{2} / R$ be the two proper, nonzero, b-divisible submodules of $K$. Let $S=A_{1} \cap A_{2}$ be the special $Q_{2}$-ring containing $R$, and let $M$ be a maximal ideal of $R$. Then

(1) $S$ bas at most two maximal ideals lying over $M$.

(2) $S_{M}$ is an b-local ring with at most two maximal ideals.

(3) $R$ is a special $Q_{2}$-ring if and only if $S$ bas exactly one maximal ideal lying over $M$ for every maximal ideal $M$ of $R$. In this case $R=S$.

Proof. (1) Suppose that $N$ is a maximal ideal of $S$ lying over $M$. With the notation of Theorem 5.2 we can assume that $N \in C_{1}$; and thus $\left(A_{1}\right)_{N}=S_{N}$ and $\left(A_{2}\right)_{N}=Q$. We have $M A_{1} \subset N A_{1} \subset N S_{N}$, and thus $M A_{1}$ is a proper ideal of $A_{1}$. Since $A_{1}$ is a compatible ring extension of $R$ by Theorem 4.5, we conclude from Theorem 1.9 that $M A_{1}$ is a maximal ideal of $A_{1}$. It follows that $M A_{1}=N A_{1}$, and hence $M A_{1} \cap S=N A_{1} \cap S=N$. This shows that there is at most one maximal ideal of $S$ in $C_{1}$ lying over $M$. Similarly there is at most one maximal ideal of $S$ in $\mathcal{C}_{2}$ lying over $M$. Therefore, $S$ has at most two maximal ideals lying over $M$.

(2) Since $S$ is integral over $R$ by Theorem 4.5, every maximal ideal of $S_{M}$ contracts in $S$ to a maximal ideal lying over $M$. Therefore by (1) $S_{M}$ has at most two maximal ideals. Suppose that $S_{M}$ does have two distinct maximal ideals. These contract in $S$ to two distinct maximal ideals $N_{1}$ and $N_{2}$. By the argument of (1) we can assume that $N_{1} \in \mathcal{C}_{1}$ and $N_{2} \in \mathcal{C}_{2}$. By Theorem 5.2, $N_{1} \cap N_{2}$ contains no nonzero prime ideal of $S$. Therefore, $N_{1} S_{M} \cap N_{2} S_{M}$ contains no nonzero prime ideal of $S_{M}$, showing that $S_{M}$ is an $b$-local ring.

(3) If $R$ is a special $Q_{2}$-ring, then $S=A_{1} \cap A_{2}=R$, and hence, of course, $S$ has only one maximal ideal lying over each maximal ideal of $R$. Conversely, suppose that $S$ has only one maximal ideal lying over each maximal ideal $M$ of $R$. Then $S_{M}$ is a quasi-local ring, and as in the proof of Theorem 5.2 we can assume that $\left(A_{1}\right)_{M}=S_{M}$ and $\left(A_{2}\right)_{M}=Q$. Therefore, $S_{M} / R_{M}$ is a divisible $R_{M}$-module. But by Theorem 4.5, $S_{M} / R_{M}$ is a torsion $R_{M}$-module of bounded order. Thus we have $S_{M}=R_{M}$. Therefore $S \subset \cap S_{M}=\bigcap R_{M}$, where $M$ ranges over all of the maximal ideals of $R$. But $\cap R_{M}=R$, and hence we have $S=R$. 
Corollary 5.4. Suppose that $R$ is a quasi-local $Q_{2}$-ring, and let $A_{1} / R$ and $A_{2} / R$ be the two proper, nonzero, $b$-divisible submodules of $K$. Then $S=A_{1} \cap A_{2}$ is an b-local, special $Q_{2}$-ring with exactly two distinct maximal ideals $N_{1}$ and $N_{2}$, and we have $A_{1}=S_{N_{1}}$ and $A_{2}=S_{N_{2}}$.

Proof. If $M$ is the maximal ideal of $R$, then $S=S_{M}$. We cannot have $S=R$, since $K$ is an indecomposable $R$-module by [8, Corollary 4.2]. Hence by Theorem 5.3, $S$ is an $b$-local special $Q_{2}$-ring with exactly two distinct maximal ideals $N_{1}$ and $N_{2}$. By Theorem 5.2, we have $A_{1}=S_{N_{1}}$ and $A_{2}=S_{N_{2}}$.

Theorem 5.5. An integral domain $R$ is an b-local, special $Q_{2}$-ring if and only if $R$ is a $Q$-ring and $R_{M} / R$ is a nonzero, $b$-divisible $R$-module for every maximal ideal $M$ of $R$. In this case $R$ bas exactly two distinct maximal ideals.

Proof. Suppose that $R$ is an $b$-local, special $Q_{2}$-ring. Since $R$ is an $b$-local ring, $R_{M} / R$ is a direct summand of $K$ by [7, Theorem 8.5] and hence is an $b$-divisible $R$-module for every maximal ideal $M$ of $R$. Because $R$ is a special $Q_{2}$-ring, $K$ is a decomposable $R$-module, and thus $R$ is not a quasi-local ring by [8, Corollary 4.2]. Therefore, $R_{M} / R$ is nonzero for every maximal ideal $M$ of $R . R$ is a $Q_{2}$-ring by Lemma 4.4 .

Conversely, suppose that $R$ is a $Q$-ring and that $R_{M} / R$ is a nonzero $b$-divisible submodule of $K$ for every maximal ideal $M$ of $R$. Then $K$ has more than one proper nonzero $b$-divisible submodule, and hence $R$ is a $Q_{2}$-ring with exactly two maximal ideals $M_{1}$ and $M_{2}$. Now $R_{M}+R_{M_{2}}=Q$ by Theorem 4.5; and, of course, $R_{M_{1}} \cap R_{M_{2}}=R$. Therefore, $R$ is a special $Q_{2}$-ring by'Theorem 4.5. By Theorem 5.2, $M_{1} \cap M_{2}$ contains no nonzero prime ideal of $R$, and thus $R$ is an $b$-local ring.

6. Noetherian domains of Krull dimension 1.

Definition. A domain is said to have Krull dimension 1 if it is not a field and if every nonzero prime ideal is a maximal ideal.

Lemma 6.1. If $R$ is a Noetherian, local domain of Krull dimension 1, then every b-reduced submodule of $K$ is a finitely generated $R$-module.

Proof. Suppose that $D$ is an $b$-reduced submodule of $K$, but $D$ is not finitely generated. Since $K$ has the descending chain condition on submodules by $[6$, Theorem 1], we can assume that $D$ is minimal with respect to the property of not being finitely generated. An $R$-module is divisible if and only if it is $b$-divisible by $[5$, Theorem 3.3] and hence there is an element $r \in R$ such that $r D \neq D$. By the minimality of $D, r D$ is finitely generated and thus has bounded order. Because $D / r D$ also has bounded order, it follows that $D$ has bounded order. Hence $s D=0$ for some nonzero element $s \in R$.

Now $D=C / R$, where $C$ is an $R$-submodule of $Q$ containing $R$. Thus we have 
$s C \subset R$, and hence $C$ and $D$ are finitely generated $R$-modules. This contradiction proves that every $b$-reduced $R$-submodule of $K$ is finitely generated.

Remarks. The following theorem was proved originally for principal ideal domains by F. K. Schmidt [14]. The present slightly more general version is proved by a trivial modification of a proof of Schmidt's theorem that was communicated to me by Barbara Osofsky.

Theorem 6.2. If $R$ is a nonlocal Noetherian domain of Krull dimension 1, then $R_{M}$ is not complete for any maximal ideal $M$ of $R$.

Proof. By localization we can assume that $R$ has exactly two maximal ideals. Suppose that $R_{M}$ is a complete ring, where $M$ is one of these maximal ideals. By [6, Theorem 4, Corollary 1] the integral closure $V$ of $R_{M}$ in $Q$ is a complete discrete valuation ring. If $S$ is the integral closure of $R$ in $Q$, then using [10, Theorem 33.2] we see that $S$ is a nonlocal principal ideal domain with a finite number of maximal ideals. But then $V$ is the localization of $S$ with respect to one of these maximal ideals. Hence we can assume without loss of generality that $R$ is a principal ideal domain.

By localization again we can assume that $R$ has exactly two maximal ideals, that these two maximal ideals can be generated by prime elements $p$ and $q$ in $R$ such that $p+q=1$, and that $R_{(q)}$ is a complete ring. If $R$ has characteristic $\neq 2$, then $X^{2}-p$ factors $(\bmod (q))$ into relatively prime factors; and if $R$ has characteristic $=2$, then $X^{3}-p$ factors $(\bmod (q))$ into relatively prime factors. Thus by Hensel's lemma [10, Theorem 30.3], $p$ is either a square or a cube in $Q$. Neither of these cases is possible, and hence $R_{M}$ is not complete for any maximal ideal $M$ of $R$.

\section{Theorem 6.3. There are no Noetherian $Q_{2}$-rings of Krull dimension 1 .}

Proof. Suppose that $R$ is a Noetherian $Q_{2}$-ring of Krull dimension 1. By Theorem 4.5 we can assume that $R$ is a special $Q_{2}$-ring. Since a Noetherian domain of Krull dimension 1 is an $b$-local ring, we see by Theorem 5.5 that $R$ has exactly two distinct maximal ideals $M_{1}$ and $M_{2}$, and that $R_{M_{1}} / R$ and $R_{M_{2}} / R$ are the two proper, nonzero, $b$-divisible submodules of $K$. Hence by Theorem 4.5, $R_{M_{1}}$ and $R_{M_{2}}$ are complete in the ir respective topologies. However, the $R_{M_{i}}{ }^{-}$ topology is the $M_{i} R_{M_{i}}$-adic topology, and thus $R_{M_{i}}$ is a complete, Noetherian local domain for $i=1,2$. But this contradicts Theorem 6.2, and hence Noetherian $Q_{2}$-rings of Krull dimension 1 do not exist.

Corollary 6.4. If $R$ is a Noetherian Q-ring of Krull dimension 1 , then $R$ is a local ring.

Proof. If $R$ is not a local ring, then $R_{M} / R$ is a proper, nonzero, $b$-divisible submodule of $K$ for every maximal ideal $M$ of $R$ by [5, Lemma 3.1]. Hence $R$ is 
a $Q_{2}$-ring by Theorem 5.5. This contradicts Theorem 6.3 , and hence $R$ is a local ring.

Definition. A commutative Noetherian local ring is said to be analytically unramified if 0 is the only nilpotent element of its completion. It is said to be analytically irreducible if its completion is an integral domain.

Suppose that $R$ is a Noetherian local domain of Krull dimension 1. Then by [11, Theorem 8], $R$ is analytically unramified if and only if its integral closure in $Q$ is a finitely generated $R$-module; whereas by [8, Theorem 7.6], $R$ is analytically irreducible if and only if every proper submodule of $K$ is finitely generated. It is easy to see then that $R$ is analytically irreducible if and only if the integral closure of $R$ in $Q$ is a discrete valuation ring that is a finitely generated $R$-module.

Corollary 6.5. Suppose that $R$ is a Noetherian Q-ring of Krull dimension 1. Then $R$ is a local ring, and is a $Q_{0}$ - or a $Q_{1}$-ring. $R$ is a $Q_{0}^{-r i n g}$ if and only if it is analytically unramified. If $R$ is a $Q_{0}$-ring, then it is actually analytically irreducible, and its integral closure in $Q$ is a discrete valuation ring that is a $Q_{0}$-ring. Furthermore, if $R$ is a $Q_{0}$-ring, then it bas characteristic 2 , and the quotient field of $H$ is a purely inseparable extension of $Q$ of degree 2.

Proof. $R$ is a local ring by Corollary 6.4 , and it is a $Q_{0}$ - or a $Q_{1}$-ring by Theorem 6.3. The integral closure of a $Q_{1}$-ring is not finitely generated by Theorem 4.3; and hence if $R$ is analytically unramified, it is a $Q_{0}$-ring. Conversely, suppose that $R$ is a $Q_{0}$-ring. Then every proper submodule of $K$ is finitely generated by Theorem 6.1, and thus $R$ is analytically irreducible. If $V$ is the integral closure of $R$ in $Q$, then $V$ is a discrete valuation ring. By Theorem 2.5, $V$ is a $Q_{0}$-ring.

Suppose that $R$ is a $Q_{0}$-ring and that the quotient field of $H$ is not a purely inseparable extension of $Q$. Then by Theorem 4.8 , there is an element $x$ in $H$ that is integral over $R$ and such that $R[x]$ is a $Q_{2}$-ring. But $R[x]$ is a finitely generated $R$-module, and hence $R[x]$ is a Noetherian domain of Krull dimension 1. But this contradicts Theorem 6.3, and hence the quotient field of $H$ is purely inseparable over $Q$. Therefore $R$ has characteristic 2 .

Corollary 6.6. If $R$ is a Noetherian local domain of Krull dimension 1 that is analytically unramified and bas a perfect residue field, then $R$ is not a $Q$-ring.

Proof. Suppose that $R$ is a $Q$-ring. Then by Corollary 6.5, $R$ is a $Q_{0}$-ring of characteristic 2. Furthermore the integral closure $V$ of $R$ in $Q$ is a discrete valuation ring; $V$ is a finitely generated $R$-module and is a $Q_{0}$-ring. The residue field of $V$ is finite-dimensional over the residue field of $R$, and hence the residue field of $V$ is perfect. Thus without loss of generality we can assume that $R$ is a discrete valuation ring. Therefore, $H$ is a complete, discrete valuation ring of characteristic 2 and perfect residue field $F$. 
By the structure theorem of I. S. Cohen [13, Theorem 2.7, Corollary], $H$ is isomorphic as a ring to $F[[X]]$, the ring of all formal power series in $X$ with coefficients in $F$ and positive integer exponents. We will identify $H$ with $F[[X]]$. Let $V$ be the set of all elements of $F[[X]]$ that are squares of elements of $F[[X]]$. Then $V$ is a ring, and since $H$ is purely inseparable over $R$ by Corollary 6.5, we have $V \subset R \subset F[[X]]$. Since $F$ is a perfect field of characteristic 2 , it is easy to see that an element of $F[[X]]$ whose exponents are all even integers is a square. Thus every element of $F[[X]]$ can be written in the form $u+v X$, where $u, v \in V$. Therefore $F[[X]]$ is a finitely generated $V$-module, and hence a finitely generated $R$-module. But $H / R$ is a nonzero divisible $R$-module, and hence we have a contradiction. Therefore, $R$ is not a $Q$-ring.

Remarks. We note that C. U. Jensen states [3, Proposition 4] that if $R$ is a Noetherian domain of Krull dimension 1 that is either nonlocal, or a local, analytically unramified ring with perfect residue field, then $\operatorname{Ext}_{R}^{1}(Q, R)$ is either 0 or has infinite dimension over $Q$. Thus Corollary 6.4 and Corollary 6.6 are only weaker versions of Jensen's more general result.

In the next section we shall present examples of a Noetherian $Q_{0}$-ring and a Noetherian $Q_{1}$-ring, both of characteristic 2 . It remains an open question whether or not there exist any Noetherian $Q_{1}$-rings of Krull dimension 1 and characteristic $\neq 2$.

Theorem 6.7. Suppose that $R$ is a Noetherian domain of Krull dimension 1 that is not complete. Then the following statements are equivalent:

(1) There is a $Q_{1}$-ring between $R$ and $Q$.

(2) The integral closure of $R$ in $Q$ is a complete, discrete valuation ring.

(3) There exists a torsion-free, cotorsion $R$-module of Q-rank 1.

(4) There exists a nonzero $R$-homomorphism of $H$ into $Q$ that is not an epimorphism.

If any of these statements are true, then $R$ is a local ring and is not analytically unramified.

Proof. $(1) \Rightarrow(4)$. Suppose that $A$ is a $Q_{1}$-ring such that $R \subset A \subsetneq Q$. By Theorem 4.3 there is a ring homomorphism of $H(A)$, the completion of $A$, into $Q$ that is not onto. By Theorem 1.12, there is a ring homomorphism of $H$ into $H(A)$. The composite of these two ring homomorphisms is a nonzero $R$-homomorphism of $H$ into $Q$ that is not onto.

$(4) \Rightarrow(3)$. Since $Q$ has homological dimension 1 as an $R$-module [5, Lemma 3.2], an $h$-reduced $R$-homomorphic image of $H$ is a cotorsion $R$-module [7, Theorem 10.1].

$(3) \Rightarrow(2)$. Let $C$ be a torsion-free, cotorsion $R$-module of $Q$-rank 1 , and let $T=\operatorname{Hom}_{R}(C, C)$. Then $T$ is isomorphic to a ring extension $S$ of $R$ in $Q$, and $S$ is complete in the $S$-topology by [7, Corollary 6.11]. Therefore, $S$ is a complete, 
Noetherian, local domain of Krull dimension 1 by [10, Theorem 33.2] and [8, Corollary 4.3]. If $V$ is the integral closure of $S$ in $Q$, then $V$ is a complete, discrete valuation ring by [6, Theorem 4, Corollary 1$]$. Now the integral closure of $R$ in $Q$ is a principal ideal domain, and $V$ is a localization of it with respect to one of its prime ideals. Hence by Theorem $6.2, V$ is the integral closure of $R$ in $Q$.

$(2) \Rightarrow(1)$. Suppose that the integral closure of $R$ in $Q$ is a complete, discrete valuation ring $V$. Then every ring between $R$ and $V$ is a Noetherian local domain of Krull dimension 1 by [10, Theorem 33.2]. Let $S / R=b(V / R)$, where $R \subset S \subset V$. By Corollary $1.10, S$ is a compatible ring extension of $R$. By [7, Theorem 10.1], $V / S$ is an $b$-reduced $R$-module; and hence by Theorem 6.1 and Theorem 1.9(2), $V / S$ is a finitely generated $R$-module. Let $x_{1}, \cdots, x_{n}$ be elements of $V$ whose images in $V / S$ generate $V / S$ as an $R$-module. Let $R^{1}=R\left[x_{1}, \ldots, x_{n}\right]$; since the $x_{i}$ 's are integral over $R, R^{1}$ is a finitely generated $R$-module. By Theorem 2.5, $R^{1}$ is not complete. Now $V=S+R^{1}$, and hence $V / R^{1} \cong S /\left(R^{1} \cap S\right)$ is a homomorphic image of $S / R$. Therefore, $V / R^{1}$ is an $b$-divisible $R$-module; and hence by Corollary 1.6, $V / R^{1}$ is an $b$-divisible $R^{1}$-module. Without loss of generality we can assume that $R=R^{1}$.

If $M$ is the maximal ideal of $R$, then $H$ is the $M$-adic completion of $R$, and thus $H$ is a Noetherian ring. The set $\left\{\operatorname{Hom}_{R}(K, D)\right\}$ where $D$ is a proper submodule of $V / R$ is a set of ideals in $H$, and hence has a maximal element $\operatorname{Hom}_{R}(K, D)$. Let $A / R=b(D)$, where $R \subset A \varsubsetneqq V$. Then by Corollary 1.3, $A / R$ is a maximal, proper, $b$-divisible submodule of $V / R$. By Corollary 1.8, $A$ is a compatible ring extension of $R$ in $Q$. Since $V / A$ is a nonzero $b$-divisible $R$-module, $A$ is not complete in the $A$-topology. Without loss of generality we can assume that $R=A$. The point of our normalizations is that now $V / R$ is a nonzero $b$-divisible $R$-module, and every proper submodule of $V / R$ is $b$-reduced (and hence finitely generated by Theorem 6.1).

In fact, $V / R$ is the only proper, nonzero, $b$-divisible submodule of $K$. For suppose that $B / R(R \subset B \varsubsetneqq Q)$ is a proper, nonzero, $b$-divisible submodule of $K$. By Corollary $1.8, B$ is a compatible ring extension of $R$. Let $\bar{B}$ be the integral closure of $B$ in $Q$. Since $V$ is integral over $R$, we have $V \subset \bar{B}$. But $V$ is a discrete valuation ring and hence $V=\bar{B}$. Therefore $B \subset V$. Since $V / R$ has no proper, nonzero, $b$-divisible submodules, we have $B / R=V / R$.

By Theorem 1.2 there is a nonzero $R$-homomorphism $f$ of $\dot{K}$ into $V / R$, and hence onto $V / R$. Let $C / R=\operatorname{Ker} f$, where $R \subset C \varsubsetneqq Q$; then $V / R \cong K / \operatorname{Ker} f \cong Q / C$. Now $C / R$ is not $b$-reduced. For if it were, $C$ would be finitely generated by Theorem 6.1 , and we could then find an $R$-homomorphism of $V / R \cong Q / C$ onto $K$. But then every proper $R$-submodule of $K$ would be finitely generated, and this is not the case. Therefore, $C / R$ is not $b$-reduced and hence $V / R \subset C / R$. By Theorem 1.9(5), $C$ is thus a $V$-module, and hence we have $C \cong V$. Thus we see that $V / R \cong Q / C \cong Q / V$. 
By Theorem 1.10 we have an exact sequence:

$$
0 \rightarrow \operatorname{Hom}_{R}(K, V / R) \rightarrow H \rightarrow H(V),
$$

where $H(V)$ is the completion of $V$. But $V$ is complete and hence $H(V) \cong V$. Using [7, Proposition 3.2] we have $\operatorname{Hom}_{R}(K, V / R) \cong \operatorname{Hom}_{R}(K, Q / V) \cong H(V) \cong V$. Thus the exact sequence $(*)$ shows that $Q$-rank $H \leq 2$. Since $R$ is not complete, we have $Q$-rank $H=2$, and thus $R$ is a $Q$-ring by Theorem 2.2. Because $K$ has exactly one nonzero, proper, $b$-divisible submodule, $R$ is a $Q_{1}$-ring. By Theorem 4.3, $R$ is not analytically unramified.

Corol lary 6.8. If $R$ is a Noetherian domain of Krull dimension 1 that is not local (or is local and analytically unramified) then $H$ is isomorpbic as an R-module to a subdirect product of copies of $Q$.

Proof. Since $H$ is torsion-free and $Q$ is an injective $R$-module, there exists for every nonzero element $x \in H$ an $R$-homomorphism $\phi_{x}$ of $H$ into $Q$ such that $\phi_{x}(x) \neq 0$. Therefore, we have an imbedding of $H$ into a direct product of copies of $Q$. By Theorem 6.7 every nonzero $R$-homomorphism of $H$ into $Q$ is an epimorphism. Thus $H$ is imbedded as a subdirect product of copies of $Q$.

Remarks. It follows from Theorem 6.2 that a complete domain cannot be a localization with respect to a maximal ideal of a nonlocal Noetherian domain of Krull dimension 1. Theorem 6.7 provides an example of a noncomplete Noetherian local domain of Krull dimension 1 that cannot be such a localization. For let $R$ be a Noetherian $Q_{1}$-ring of Krull dimension 1 (such a ring exists as we shall see in the next section). Then by Theorem 6.7 there does not exist a nonlocal Noetherian domain $S$ of Krull dimension 1 with quotient field $Q$ such that $S \subset R \subset Q$.

Theorem 6.9. Suppose that $R$ is a Noetberian domain of Krull dimension 1 and that $A$ is an $R$-module. Then $\operatorname{Hom}_{R}(A, R)=0$ and $\operatorname{Ext}_{R}^{1}(A, R)$ is torsionfree if and only if $A$ is a divisible R-module.

Proof. Suppose that $\operatorname{Hom}_{R}(A, R)=0$ and that $\operatorname{Ext}_{R}^{1}(A, R)$ is torsion-free. Let $M$ be a maximal ideal of $R$. Then we have an exact sequence:

$$
0 \rightarrow \operatorname{Hom}_{R}(M A, R) \stackrel{a}{\longrightarrow} \operatorname{Ext}_{R}^{1}(A / M A, R) \rightarrow \operatorname{Ext}_{R}^{1}(A, R) .
$$

Since $\operatorname{Ext}_{R}^{1}(A / M A, R)$ is a torsion $R$-module and $\operatorname{Ext}_{R}^{1}(A, R)$ is torsion-free, w.e see that $\alpha$ is an isomorphism. But $\operatorname{Hom}_{R}(M A, R)$ is a torsion-free $R$-module, and thus $\operatorname{Ext}_{R}^{1}(A / M A, R)=0$. Now $\operatorname{Ext}_{R}^{1}(A / M A, R) \simeq \operatorname{Hom}_{R}(A / M A, K)$, and thus $\operatorname{Hom}_{R}(A / M A, K)=0$. Because $A / M A$ is a direct sum of copies of $R / M$ and $\operatorname{Hom}_{R}(R / M, K)=M^{-1} / R \neq 0$, we see that $A=M A$. Let $r$ be a nonzero element of $R$ that is not a unit in $R$. Then $R r$ contains a finite product of maximal ideals of $R$, and hence $A=r A$. Thus $A$ is a divisible $R$-module. 
Conversely, suppose that $A$ is a divisible $R$-module. Then clearly $\operatorname{Hom}_{R}(A, R)=0$. Let $r$ be a nonzero element of $R$. Then we have an exact sequence:

$$
0 \rightarrow T \rightarrow A \stackrel{r}{\longrightarrow} A \rightarrow 0
$$

where $T$ is a torsion $R$-module. Hence we have an exact sequence:

$$
0 \rightarrow \operatorname{Ext}_{R}^{1}(A, R) \stackrel{r}{\longrightarrow} \operatorname{Ext}_{R}^{1}(A, R) \rightarrow \operatorname{Ext}_{R}^{1}(T, R) .
$$

Therefore, $\operatorname{Ext}_{R}^{1}(A, R)$ is a torsion-free $R$-module.

Corollary 6.10. Let $R$ be a Noetherian domain of Krull dimension 1. If $A$ is an $R$-module such that $\operatorname{Hom}_{R}(A, R)=0$ and $\operatorname{Ext}_{R}^{1}(A, R) \cong Q$, then $A \cong Q$.

Proof. By Theorem 6.9, $A$ is a divisible $R$-module and hence $A / t(A)$ is divisible. Since $Q$-rank $(A / t(A))=1$ by Theorem 2.1 , we see that $A / t(A) \cong Q$. Let $T=t(A)$; then by [5, Theorem 3.3], $A \cong T \oplus Q$. Since $R$ is a $Q$-ring by Theorem 2.1, it follows that $\operatorname{Ext}_{R}^{1}(T, R)=0$. Now $\operatorname{Ext}_{R}^{1}(T, R) \cong \operatorname{Hom}_{R}(T, K)$; and hence it is sufficient to prove that if $T$ is a torsion divisible $R$-module such that $\operatorname{Hom}_{R}(T, K)=0$, then $T=0$.

By [5, Lemma 3.1] we have $\operatorname{Hom}_{R}(T, K) \cong \Sigma_{M} \bigoplus \operatorname{Hom}_{R_{M}}\left(T_{M}, K_{M}\right)$, where $M$ ranges over all of the maximal ideals of $R$. Thus without loss of generality we can assume that $R$ is a local ring with maximal ideal $M$. Let $E$ be the injective envelope of $R / M$. If $T \neq 0$, then $\operatorname{Hom}_{R}(T, E) \neq 0$ and we can take a nonzero element $f \in \operatorname{Hom}_{R}(T, E)$. Let $D=\operatorname{Im} f$; then $D$ is an Artinian divisible $R$-module and $\operatorname{Hom}_{R}(D, K)=0$. But in a paper, 1-dimensional Cohen-Macaulay rings (SpringerVerlag No. 327) we have proved that if $D$ is a nonzero Artinian divisible $R$-module over a 1 -dimensional local domain, then $\operatorname{Hom}_{R}(D, K) \neq 0$. This contradiction proves that $T=0$, and thus $A \cong Q$.

7. Examples and counterexamples.

I. We first present some examples of rings that cannot be $Q$-rings, or $Q_{i}$-rings, for various $i=0,1,2$.

(1) If $Q$ does not have a quadratic field extension, then $R$ cannot be a $Q_{0}$ ring by Corollary 4.7 .

(2) If $R$ is integrally closed, then $R$ cannot be a $Q_{1}$-ring by Theorem 4.3.

(3) If $R$ is a quasi-local ring, then $R$ cannot be a special $Q_{2}$ ring by Theorem 5.5.

(4) If $R$ is a quasi-local, integrally closed ring, then $R$ cannot be a $Q_{2}$-ring by (3) and Theorem 4.5 .

(5) If $R$ is a quasi-local, integrally closed ring with algebraically closed quotient field, then $R$ cannot be a $Q$-ring by (1), (2), and (4).

(6) If $R$ is a nonlocal Noetherian domain of Krull dimension 1, then $R$ cannot be a $Q$-ring by Corollary 6.4 . 
(7) If $R$ is a Noetherian local domain of Krull dimension 1 , and if $R$ has characteristic $\neq 2$ and is analytically unramified, then $R$ cannot be a $Q$-ring by Corollary 6.5. (See also [3, Theorem 1].)

II. We shall now present examples of all three different kinds of $Q$-rings.

(1) An example of a Noetberian $Q_{0}$-ring of Krull dimension 1 (and characteristic 2).

This example is due to Nagata [10, Example 3.3, p. 207]. Let $F$ be a field of characteristic 2 such that $\left[F: F^{2}\right]=\infty$. If we let $V^{*}=F[[X]]$, then $V^{*}$ is a complete, discrete valuation ring. Let $W$ be the ring consisting of those power series in $V^{*}$ whose coefficients generate a finite-dimensional extension of $F^{2}$. Then $W$ is a discrete valuation ring and $V^{*}$ is the completion of $W$. Let $L^{*}$ be the quotient field of $V^{*}$ and $S$ the quotient field of $W$. Since $\left[F: F^{2}\right]=\infty$, we see that $W \neq V^{*}$, and consequently $S \neq L^{*}$.

Let $\left\{x_{\lambda}\right\}, \lambda \in \Lambda$, be a maximal 2 -basis of $L^{*}$ over $S$. Fix $\lambda_{0} \in \Lambda$, and let $L$ be the field generated by $S$ and all of the $x_{\lambda}$ 's except $x_{\lambda_{0}}$. We have $\left[L^{*}: L\right]=2$; for if $v \in V^{*}$, then $v^{2} \in F^{2}[[X]] \subset W \subset L$. We let $V=V^{*} \cap L$; then $V$ is a discrete valuation ring and its quotient field is $L$.

$V^{*} / V$ is clearly a torsion-free $W$-module, and being a homomorphic image of $V^{*} / W$ it is divisible over $W$ as well. Thus $V^{*}$ is the completion of $V$ in the $W$ topology. Hence by Corollary 1.6, $V^{*}$ is the completion of $V$ in the $V$-topology. Since $L$-rank $V^{*}=2$, we see that $V$ is a $Q$-ring by Theorem 2.2 . Because the $V$ submodules of $L / V$ are linearly ordered, $V$ is a $Q_{0}$-ring.

(2) An example of a Noetberian $Q_{1}$-ring of Krull dimension 1 and characteristic 2.

With the notation of example (1), choose $x \in V^{*}$ such that $x \notin V$. If we let $R=V[x]$, then by Theorem $4.8, R$ is a $Q_{1}$-ring. Since $R$ is a finitely generated $V$-module, $R$ is a Noetherian domain of Krull dimension 1 .

(3) An example of a $Q_{0}$-ring of characteristic 0 .

This example was communicated to me by C. U. Jensen. Let $p$ be a nonzero prime integer; for the sake of simplicity we will assume that $p \equiv 1(\bmod 4)$ (although any other nonzero prime would do just as well). Let $\hat{Z}_{p}$ be the $p$-adic integers, and $Q_{p}$ the quotient field of $\hat{Z}_{p}$. Let $v$ be the $p$-adic valuation on $Q_{p}$. Then $v$ can be extended to the algebraic closure $\bar{Q}_{p}$ of $Q_{p}$. It is well known that $\bar{Q}_{p}$ is not complete with respect to $v$, but that the completion $L$ of $\bar{Q}_{p}$ is an algebraically closed field. Now $L$ is algebraically isomorphic to the field of complex numbers $C$, for $L$ is algebraically closed and of transcendence degree $2^{\mathrm{NO}_{0}}$ over the rational numbers. Thus we have a nondiscrete rank one valuation $v$ on $C$ whose restriction to the rational numbers is the $p$-adic valuation.

Let $V^{*}$ be the valuation ring of $v$ in $C$; that is, $V^{*}=\{x \in C \mid v(x) \geq 0\}$; then $V^{*}$ is a complete, nondiscrete rank one valuation ring. Let $R$ be the field of real numbers, and let $V=V^{*} \cap \mathscr{R}$; then $V$ is a nondiscrete rank one valuation ring. 
Now $V$ is not complete; for if it were, since $Z_{p} \subset V, V$ would contain $\hat{Z}_{p}$, the completion of $Z_{p}$ in the p-adic valuation. But by Hensel's lemma $(-1)$ is a square in $\hat{Z}_{p}$ and -1 is not a square in $R$. Thus $V$ is not complete, and hence $V^{*}$ is the completion of $V$. Since $R$-rank $V^{*}=2$, we see by Theorem 2.2 that $V$ is a $Q$-ring. Because the $V$-submodules of $R / V$ are linearly ordered, $V$ is a $Q_{0}$-ring.

(4) An example of a $Q_{2}$-ring of characteristic 0 .

With the notation of (3), let $S$ be the integral closure of $V$ in $C$. Then by Corollary $4.10, S$ is a special $Q_{2}$-ring.

(5) An example of an b-local special $Q_{2}$-ring (of characteristic 0 ) that is a ring of type I.

In [9] we have defined an integral domain $R$ to be a ring of type $I$ if $R$ is an $b$-local ring with exactly two maximal ideals $M_{1}$ and $M_{2}$, and $R_{M_{1}}$ and $R_{M_{2}}$ are maximal valuation rings. By [7, Lemma 8.1] we have $R_{M_{1}}+R_{M_{2}}=Q$; and, of course, we have $R_{M_{1}} \cap R_{M_{2}}=R$. Since a maximal valuation ring is a complete ring, $R_{M_{i}}$ is complete in the $R$-topology by Theorem 1.5. Therefore, a ring of type $I$ is an $b$-local special $Q_{2}$-ring.

The following example was communicated to me by Barbara Osofsky. Let $C$ be the field of complex numbers and $V$ the ring of power series in an indeterminate $X$ with coefficients in $C$, and exponents that are well-ordered sequences of rational numbers. Then $V$ is a maximal valuation ring. We let $Q$ be the quotient field of $V$, and define an automorphism $\sigma$ of $Q$ by keeping the elements of $C$ fixed and sending $X$ into $1-X$. We let $W=\sigma(V)$, and $R=V \cap W$. Then $R$ is a ring of type $I$, and hence $R$ is an $b$-local, special $Q_{2}$-ring.

\section{REFERENCES}

1. H. Cartan and S. Eilenberg, Homological algebra, Princeton Univ. Press, Princeton, N. J., 1956. MR 17, 1040.

2. C. U. Jensen, On the structure on $\operatorname{Ext}_{R}^{1}(A, R)$, Colloquia Math. Soc. János Bolyai, 6 Rings, modules and radicals, Keszthely (Hungary), (1971), 215-226.

3. $\longrightarrow$ On $\operatorname{Ext}_{R}(A, R)$ for torsion-free A, Bull. Amer. Math. Soc. 78 (1972), 831-834.

4. D. M. Kan and G. W. Whitehead, On the realizability of singular cohomology groups, Proc. Amer. Math. Soc. 12 (1961), 24-25. MR 23 \#A647.

5. E. Matlis, Divisible modules, Proc. Amer. Math. Soc. 11 (1960), 385-391. MR 22 $\# 6839$.

6. - Some properties of Noetherian domains of dimension 1, Canad. J. Math. 13 (1961), 569-586. MR 24 \#A1288.

7. Cotorsion modules, Mem. Amer. Math. Soc. No. 49 (1964). MR 31 \#2283.

8. - Decomposable modules, Trans. Amer. Math. Soc. 125 (1966), 147-179.

MR 34 \#1349.

9. —_ Rings of type I, J. Algebra 23 (1972), 76-87. 
10. M. Nagata, Local rings, Interscience Tracts in Pure and Appl. Math., no. 13, Interscience, New York, 1962. MR 27 \#5790.

11. D. G. Northcott, General theory of one dimensional local rings, Proc. Glasgow Math. Assoc. 2 (1956), 159-169. MR 17, 938.

12. R. J. Nunke and J. J. Rotman, Singular cohomology groups, J. London Math. Soc. 37 (1962), 301-306. MR $25 \# 3975$.

13. O. Zariski and P. Samuel, Commutative algebra. Vol. II, University Series in Higher Math., Van Nostrand, Princeton, N. J., 1960. MR 22 \#11006.

14. F. K. Schmidt, Mehrfach perfekte Körper, Math. Ann. 108 (1933), 1-25.

DEPARTMENT OF MATHEMATICS, NORTHWESTERN UNIVERSITY, EVANSTON, ILLINOIS 60201 